\title{
Mussel-Inspired Gold Nanoparticle and PLGA/ L-Lysine-g-Graphene Oxide Composite Scaffolds for Bone Defect Repair
}

\author{
Chuan Fu (D) \\ Yikun Jiang' \\ Xiaoyu Yang' \\ Yu Wang ${ }^{2}$ \\ Wei $\mathrm{Ji}^{3}$ \\ Guoliang Jia' \\ 'Department of Orthopaedic Surgery, \\ The Second Hospital of jilin University, \\ Changchun, I 3004I, People's Republic of \\ China; ${ }^{2}$ Key Laboratory of Polymer \\ Ecomaterials, Changchun Institute of \\ Applied Chemistry, Chinese Academy of \\ Sciences, Changchun, I30022, People's \\ Republic of China; ${ }^{3}$ School of Life Science \\ and Technology, Changchun University of \\ Science and Technology, Changchun, \\ 130022, People's Republic of China
}

Correspondence: Guoliang Jia

Department of Orthopaedic Surgery, The Second Hospital of Jilin University,

Changchun, I3004I, People's Republic of China

Tel +86 I5754306089

Email jiaguoliang@jlu.edu.cn
Purpose: Insufficient biological activity heavily restricts the application and development of biodegradable bone implants. Functional modification of bone implants is critical to improve osseointegration and bone regeneration.

Methods: In this study, L-lysine functionalized graphene oxide (Lys-g-GO) nanoparticles and polydopamine-assisted gold nanoparticle (AuNPs-PDA) coatings were applied to improve the biological function of PLGA scaffold materials. The effects of Lys-g-GO nanoparticles and AuNPs-PDA functionalized coatings on the physicochemical properties of PLGA scaffolds were detected with scanning electron microscopy (SEM), contact angle measurement, and mechanical testing instruments. In vitro, the effects of composite scaffolds on MC3T3-E1 cell proliferation, adhesion, and osteogenic differentiation were studied. Finally, a radial defect model was used to assess the effect of composite scaffolds on bone defect healing.

Results: The prepared AuNPs-PDA@PLGA/Lys-g-GO composite scaffolds exhibited excellent mechanical strength, hydrophilicity and antibacterial properties. In vitro, this composite scaffold can significantly improve osteoblast adhesion, proliferation, osteogenic differentiation, calcium deposition, and other cell behaviour. In vivo, this composite scaffold can significantly promote the new bone formation and collagen deposition in the radial defect site and presented good biocompatibility.

Conclusion: The combination of bioactive nanoparticles and surface coatings shows considerable potential to enhance the osseointegration of bone implants.

Keywords: gold nanoparticles, graphene oxide, bone defect, poly(dopamine), PLGA, L-lysine

\section{Introduction}

With the development of tissue engineering technology, concern regarding degradable polymer scaffolds for bone defect treatment is increasing. Currently, a large number of natural materials and synthetic polymers have been prepared as bone implants and applied in the treatment of bone defects, such as gelatin, chitosan, polypeptide, polylactic acid and PLGA. ${ }^{1-5}$ Among many degradable polymer materials, PLGA is the most widely used because of its good biocompatibility, degradability and adjustable mechanical properties. ${ }^{6}$ Many studies have adopted PLGA to prepare bone tissue engineering scaffolds and applied them to treat bone defects. ${ }^{7,8}$ However, PLGA material has some disadvantages, such as hydrophobic surfaces, deficient osteogenic induction properties, and others. ${ }^{9,10}$ Therefore, many strategies have been employed to 


\section{Graphical Abstract}

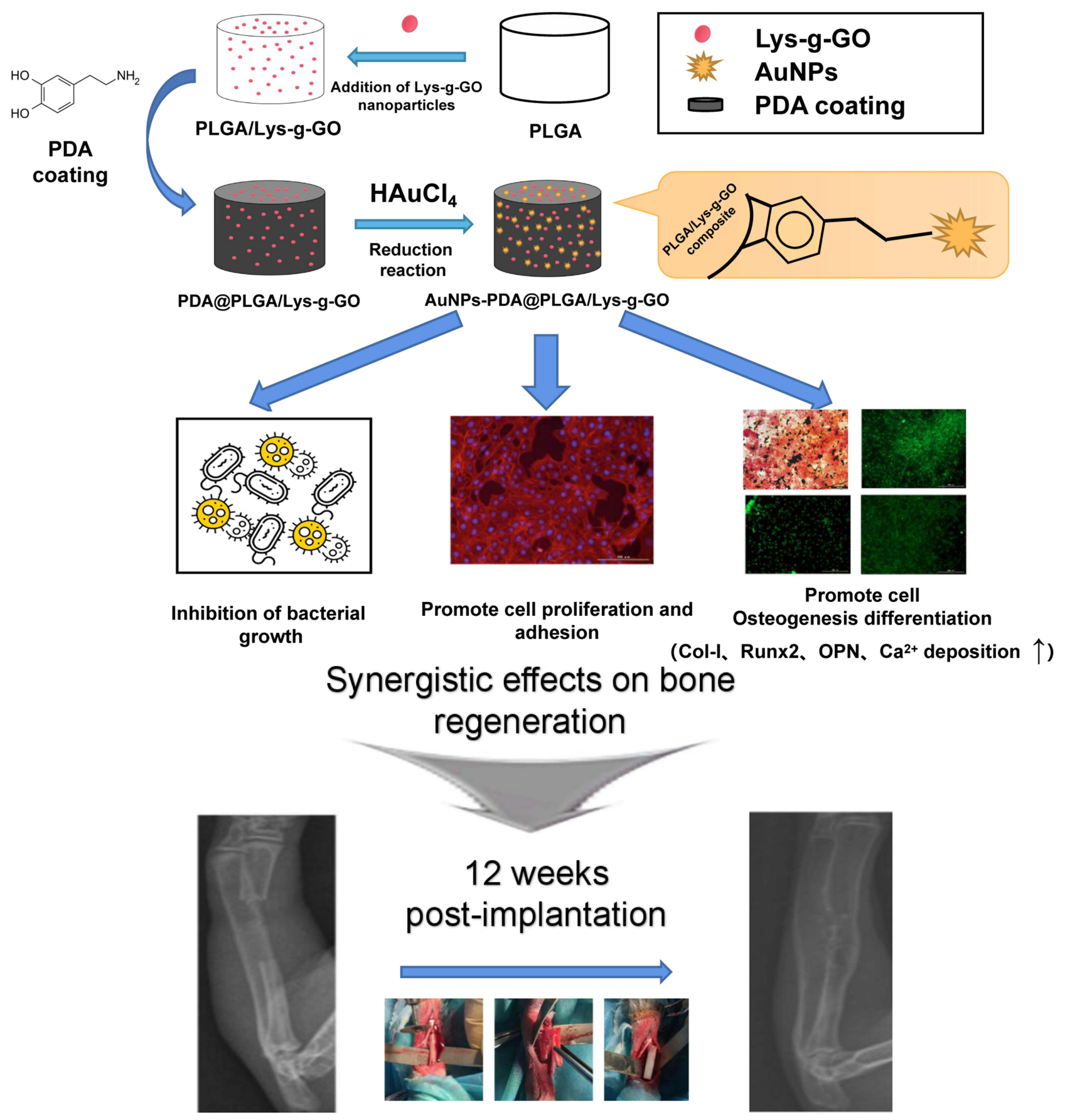

improve the bone repair ability of PLGA by function modification. ${ }^{11}$ A common modification is to blend some bioactive nanoparticles with PLGA to prepare composites, such as hydroxyapatite, graphene oxide, carbon nanotubes, and others. ${ }^{12-14}$ These nanoparticles can significantly improve scaffolds' mechanical strength, regulate cell function, and facilitate bone cell growth and fusion of the material with the surrounding bone tissue. This strategy can effectively improve the bone repair capacity of PLGA, serving as a solution to overcome the defects of a single material. 
In recent years, graphene oxide (GO) has been widely used in the functional modification of polymer materials due to its excellent physical and chemical properties. The particular surface structure and abundant functional groups provided GO with many excellent properties, such as good hydrophilicity, antibacterial properties, dispersibility, and mechanical strength. ${ }^{15-18}$ Moreover, many studies have demonstrated that GO have strong osteogenic induction ability. ${ }^{19,20}$ Some studies have discovered that GO can promote osteogenic differentiation by increasing cytoskeletal tension. ${ }^{21}$ Furthermore, the strong protein adsorption capacity of GO can attract the nutrients required for cell growth on the material surface, creating a better living environment. ${ }^{22}$ More importantly, the abundant oxygencontaining active groups such as carboxyl groups, hydroxyl groups, and epoxy groups, facilitate modification of the GO surface with bioactive factors to improve biological properties. Many studies have employed various active substances of amino acids, growth factors, drugs, and others, to further modify the surface of GO to improve the bone repair ability of composite materials. ${ }^{23-25}$ Although the addition of bioactive nanoparticles can effectively enhance the bone repair ability of polymer materials, this method still has some deficiencies, such as uneven surface distributions of nanoparticles. To maximize the bone repair ability of bone implants, many studies have used other modification methods combined with bio-active nanoparticles to enhance the biological properties of bone implants. For example, Gao et al uses both hydroxyapatite nanoparticles and gelatin coatings to improve the biological activity of PLGA materials, and the results show that the combination of these two modification methods can effectively enhance cell proliferation and osteogenic differentiation ability. ${ }^{6}$ Yang et al modified PLGA nanofibres by cellulose and polydopamine coating at the same time, and the results showed that the composite nanofibre surface had good applicability for cell adhesion, proliferation and growth. ${ }^{26}$ Furthermore, the introduction of cellulose can effectively accelerate the deposition rate of the polydopamine coating. These studies have demonstrated that different modification methods can simultaneously improve the function of scaffold materials to achieve complementary advantages, and this kind of multi-component composite has broad application prospects in the field of bone repair. In addition to blending with bioactive nanoparticles, surface modification is another classic strategy to improve PLGA material performance. Grafting or coating scaffold surfaces with bioactive substances, such as gelatin, metal nanoparticles, hydroxyapatite, and others, can effectively regulate cell functions effectively. ${ }^{27-29}$ Many studies have combined different material modification methods to maximize the bone repair ability of scaffold materials.

Gold nanoparticles (AuNPs) have attracted considerable attention in the field of bone repair due to their advantageous properties in biomedical applications such as good biocompatibility, osteogenic inducibility and easy functionalization. ${ }^{30-32}$ Recently, some studies have found that AuNPs could effectively promote the osteogenic differentiation and mineralization of cells, and ALP activity, and the expression of osteoblast-related genes (Runx-2, OPN, OCN) were significantly enhanced after cell contact with AuNPs. ${ }^{33-35}$ Other studies suggested that AuNPs could stimulate cells by regulating the (MPAK) -p38 and ERK/ MAPK pathway, thus inducing cell osteogenesis. ${ }^{36,37}$ A recent clinical trial suggested that surface modification of titanium implants with AuNPs can promote bone interface formation and bone regeneration. ${ }^{38}$ In addition, AuNPs can effectively regulate the behavior of osteoblasts and also modify the osteoclast formation pathway. Therefore, these features render AuNPs very suitable for functional modification of bone repair materials. In the application process of gold nanoparticles, their concentration, properties, morphology and synthesis methods have a very important effect on their biological applications. ${ }^{39,40}$ However, the conventional blending method of AuNPs has some limitations with respect to enhancing the properties of materials. Effective blending requires AuNPs at high concentrations, which will induce biological toxicity and reduce the biological activity of bone repair materials. Therefore, many studies prefer to use coating technology to introduce AuNPs into bone repair materials, which can not only maintain effective contact between cells and AuNPs but also avoid toxicity generation to a large extent. At present, several methods are commonly been used to apply AuNPs coatings on the surfaces of materials, such as sputtering coating and physical/chemical vapour deposition; however, these methods generally require complex instrumentation. In recent years, the polydopamine reduction has become a popular method to prepare metal nanoparticle coatings. Dopamine can oxidize and autopolymerize in oxygen and alkaline environments, forming polydopamine (PDA) coatings on most solid substance surfaces. ${ }^{41}$ The adhesion and reducibility of polydopamine can reduce gold ions to AuNPs, and facilitate their adherence to the surface of a material. Furthermore, the PDA coating can increase the material surface wettability, and 
accelerate hydroxyapatite formation, which can further improve the bone repair ability of the material. Thus, polydopamine-assisted AuNPs functionalized coating combined with bioactive nanoparticles is a superior choice to improve bone implant performance and warrants further investigation.

In this study, we modified GO with L-lysine (Lys) by chemical grafting, and blended Lys-g-GO with PLGA to prepare composite porous scaffolds (PLGA/Lys-g-GO). Then, we applied AuNPs-PDA functionalized coatings to the scaffold surface with a polydopamine as the reducing agent. The physical and chemical properties of the scaffolds were characterized by SEM, contact angle measurement, mechanical testing and antibacterial experiments. We also studied the effects of AuNPs@PLGA/Lys-g-GO composite scaffolds on cell proliferation, adhesion, osteogenic differentiation, and new bone regeneration. This research aims to improve the biological activity of PLGA through the above material modification methods and prepare composite materials with an excellent bone repair ability for bone defect repair.

\section{Materials and Methods}

A detailed schematic diagram of the scaffold preparation is shown in Figure 1.

\section{Materials}

PLGA (molecular weight=100,000, LA/GA=75/25) was obtained from ChangchunSinoBiomaterials Co., Ltd. GO was purchased from Chengdu Organic Chemicals Co. Ltd,

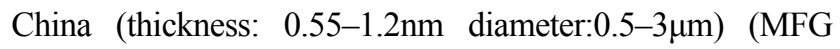
Code 021519). L-lysine was purchased from Beijing Chemical Reagent Co., Ltd, China (Product number
62016734). $\mathrm{HAuCl}_{4} \cdot 3 \mathrm{H}_{2} \mathrm{O}$ (CAS number 16961-25-4), Cetylpyridinium chloride (CPC) (CAS number 6004-24-6) and dopamine hydrochloride (CAS number 62-31-7) was purchased from Aladdin Reagents Co., Ltd, China. N-(3-Dimethylaminopropyl)-N'-ethylcarbodiimide hydrochloride (EDC) (CAS number 25952-53-8), N-Methylpyrrolidone (NMP) (CAS number 872-50-4), 3-(4,5-dimethyl-2-thiazolyl)-2, 5-diphenyl-2-H-tetrazolium bromide (MTT) (CAS number 298-93-1), 4,6-diamino-2-phenylindole (DAPI) (CAS number 28718-90-3) and rhodaminephalloidine (Product number P1951) were purchased from Sigma-Aldrich (USA). The reagents for cell experiments were purchased from Sigma-Aldrich (USA). Hematoxylin and eosin (H\&E) (Product number G1120), Masson trichrome staining (Product number G1340) and Sirius Red kits (Product number S8060) were purchased from Solarbio Co., Ltd, China.

\section{Preparation and Characterization of Lys-g-GO}

L-lysine was grafted to the surface of GO by chemical grafting. In brief, $100 \mathrm{mg} \mathrm{GO}$ was first dispersed in $50 \mathrm{~mL}$ deionized water followed by continuous ultrasound for 2 h. Subsequently, $0.25 \mathrm{~g}$ EDC was added to the above solution to activate the carboxyl group of the GO. Then, $0.3 \mathrm{~g} \mathrm{~L}$-lysine powder was added to the solution system, and stirred at room temperature for $24 \mathrm{~h}$. After the reaction is complete, the mixed solution is centrifuged by a centrifuge $(8000 \mathrm{rpm})$ (eppendorf, Centrifuge $5427 \mathrm{R}$, GER), and the resulting precipitate is rinsed repeatedly with deionized water. Finally, the Lys-g-GO were obtained with vacuum drying. Fourier transform infrared spectra

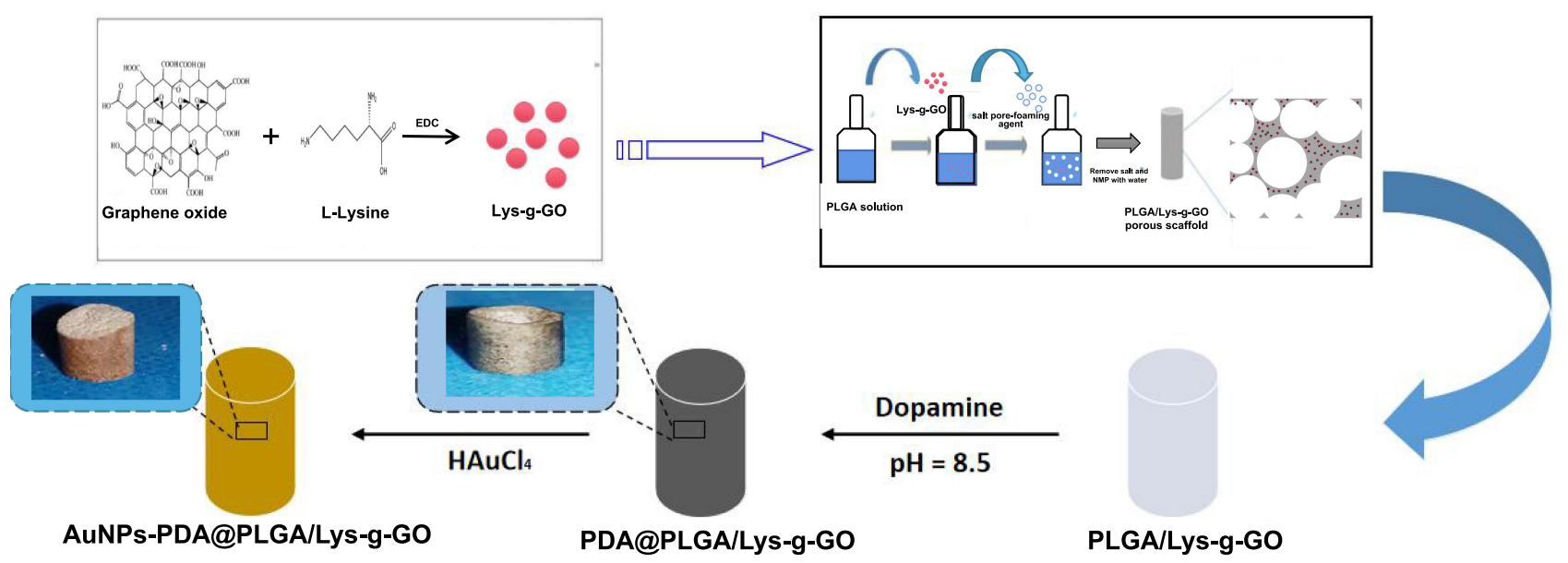

Figure I A brief schematic drawing of the preparation of AuNPs-PDA@PLGA/Lys-g-GO composite scaffolds. 
(FTIR, Perkin Elmer, 1730, USA) were used to identify the crosslinking between L-lysine and GO. The surface structures of Lys-g-GO were observed by transmission electron microscopy (TEM, FEI, Tecnai G2 F20 S-TWIN, USA).

In order to compare the effects of GO and Lys-g-GO on the physicochemical properties of PLGA materials, we added GO and Lys-g-GO into PLGA respectively, and made the composite material into a film sheet (the ratio of PLGA to nanoparticles is 99: 1). Then, the surface hydrophilicity of composite samples was studied by water contact angle tester (Kruss, DSA 10, GER). The protein adsorption properties of PLGA, PLGA/GO and PLGA/Lys-g-GO were analysed by performing a bicinchoninic acid (BCA) protein assay (Abcam, ab207003) using BSA as a model protein. Briefly, the samples were added into $4 \mathrm{~mL}$ of BSA solution $(2 \mathrm{mg} /$ $\mathrm{mL}$ ), and liquid samples were taken to analyse the solution BSA concentration after $1 \mathrm{~h}$. The amount of BSA loaded on the samples was determined by the reduction in BSA concentration.

\section{Fabrication of PLGA/Lys-g-GO Composite Scaffolds}

A preparation process combining phase inversion and particle leaching was used to prepare PLGA/Lys-g-GO composite scaffolds. Briefly, 2 g PLGA was dissolved in $10 \mathrm{~mL}$ NMP followed by the addition of $20 \mathrm{mg}$ Lysg-GO. After the above mixed solution is fully stirred, pore-foaming agent (Sodium chloride granule, 220 450 $\mu \mathrm{m})$ were added to the solution, and the mixture is poured into cylindrical mould and frozen. After the mixed solution is completely frozen, the cylindrical mould was immersed in deionized water for 3 days, and the deionized water is changed every 8 hours. After the NMP solvents and sodium chloride granule in the mixed solution were completely replaced by water, the solidified scaffold materials were freeze-dried and stored in a drying oven.

\section{PDA-Assisted Immobilization of AuNPs Onto PLGA/Lys-g-GO Composite Scaffolds}

In this study, the surface of the scaffold material was functionally treated with polydopamine, and gold nanoparticles were reduced and loaded on the surface of the scaffold through the reducibility of polydopamine. ${ }^{42,43}$ The operation process was performed as followed. First,
PLGA/Lys-g-GO composite scaffolds were immersed in dopamine solution $(2 \mathrm{mg} / \mathrm{mL}, \mathrm{pH} 8.5)$. After $24 \mathrm{~h}$, the PDA modified scaffold (PDA@PLGA/Lys-g-GO) was washed with deionized water to remove unattached dopamine molecules. Then, the scaffold was immersed in gold ion solution $(2.0 \mathrm{mM})$ overnight at room temperature. The AuNPs coating was constructed on the surface of the scaffold by the reducibility of PDA. Finally, The AuNPsPDA@PLGA/Lys-g-GO scaffolds were then washed with distilled water for to remove the unattached AuNPs. At the same time, four groups scaffold materials were prepared as control group, including pure PLGA scaffolds, PLGA/Lysg-GO scaffolds, PDA-modified PLGA scaffolds (PDA@PLGA) and PDA-assisted AuNPs-coated PLGA scaffolds (AuNPs-PDA@PLGA).

\section{Composite Scaffolds Characterization}

The composite scaffolds were cut off and the middle cross section was exposed. Then, a scanning electron microscope (SEM, Zeiss, EVO 25, GRE) was used to observe the microstructure of the composite scaffolds. The composite scaffold samples were prepared into cylinders with a diameter of $8 \mathrm{~mm}$ and a height of $25 \mathrm{~mm}$, and the mechanical properties of the composite scaffolds was detected with a multifunctional mechanical tester (Instron, Instron 1121, UK). The hydrophilicity of samples was detected by water contact angle tester (Kruss DSA 10, GER). X-ray photoelectron spectroscopy (XPS, Kratos, JPN) and X-ray diffraction (XRD, Bruker. D8 ADVANCE, GER) were used to detect the elemental composition and crystalline structure of composite scaffolds.

\section{Determination of Antibacterial Activity} Staphylococcus aureus (S. aureus, ATCC25923) and Escherichia coli (E. coli, ATCC25922) were used as strains to evaluate the antibacterial properties of composite scaffolds. All bacterial strains were provided by the Experimental Center of the Second Hospital of Jilin University. Briefly, composite scaffolds were first immersed in $75 \%$ ethanol solution and sterilized with UV. The sterilized scaffolds were then rinsed repeatedly with PBS to remove residual ethanol. The bacterial concentration was adjusted to $4 \times 10^{4} \mathrm{CFU} / \mathrm{mL}$ and cultured in a constant temperature incubator at $37^{\circ} \mathrm{C}$ for $2 \mathrm{~h}$. Then, the bacterial was co-cultured with the sterilized composite scaffolds. After $12 \mathrm{~h}, 150 \mu \mathrm{L}$ bacterial solution was transferred to a 96-well plate, and the absorbance value at 600 $\mathrm{nm}$ was measured by multifunctional microplate scanner 
(TECAN, Infinite M200, Swit). In order to further observe the antibacterial properties of composite scaffolds, $60 \mu \mathrm{L}$ of bacterial liquid was absorbed and diluted. Then, $30 \mu \mathrm{L}$ diluted bacterial solutions was evenly spread onto an LB agar plate and incubated at $37^{\circ} \mathrm{C}$ overnight. Finally, the colonies in the LB agar plate were photographed. Previous studies have proved that PLGA materials have no obvious antibacterial properties, so PLGA samples were selected as the control group.

\section{Cell Adhesion and Proliferation Assay}

Mouse pre-osteoblast MC3T3-E1 cells (Shanghai Institute of Cell Biology, China) were used as model cells to evaluate the bioactivity of the composite scaffold. Cells were cultured in high-glucose DMEM medium with $10 \%$ foetal bovine serum, $63 \mathrm{mg} / \mathrm{L}$ penicillin and $100 \mathrm{mg} / \mathrm{L}$ streptomycin at $37^{\circ} \mathrm{C}$ with $5 \% \mathrm{CO}_{2}$, and the culture medium was refreshed every 2 days. For the cell proliferation examination, the composite scaffold was immersed in $75 \%$ ethanol solution and sterilized by UV. Then, the fully sterilized composite scaffold was rinsed repeatedly with PBS and soaked in cell medium. Finally, the cells were seeded at composite scaffold in the 24-well plate at a density of $2.5 \times 10^{4} /$ well. On days 1,3 , and 7 , MTT assays were used to determine cell proliferation on different composite scaffold. At each time point, $80 \mu \mathrm{L}$ MTT solution ( $5 \mathrm{~g} \mathrm{~L}^{-1}$ in PBS) was added to the culture well, and incubated for $4 \mathrm{~h}$. After incubation, the medium was removed and $600 \mu \mathrm{L}$ of acidified isopropanol was added to solubilize the converted dye. Finally, $150 \mu \mathrm{L}$ dissolved liquid was transferred to a new 96-well plate, and the absorbance value at $540 \mathrm{~nm}$ was determined by multifunctional microplate scanner (TECAN, Infinite M200, Swit).

To evaluate cell adhesion, the adherent cells cultured on various films (PLGA, PDA@PLGA, AuNPs-PDA @ PLGA, PLGA/Lys-g-GO and AuNPs-PDA@PLGA/ Lys-g-GO) were fixed with $4 \%$ paraformaldehyde after a 3-day culture. Subsequently, cells were washed by PBS to remove paraformaldehyde, and the cytoskeleton and nucleus were stained by phalloidin (red) and DIPI (blue), respectively. Finally, the adhesion cells on the surface of different materials were observed under fluorescence microscope (Nikon, E80i, Japan).

\section{Cell Mineralization}

Calcium deposition in cells growing on composite scaffold was detected by Alizarin red (ARS) staining. Briefly, the medium was removed, and cell was fixed with paraformaldehyde after 21 day culture. After the cells were fixed, the cells/scaffold samples were rinsed with PBS, and 40mM ARS (Solarbio, G8550) was added and incubated for $30 \mathrm{~min}$ at room temperature. Then, the cells/ scaffold samples were washed with distilled water for 3 times, ARS-stained samples were treated with $10 \%(\mathrm{w} / \mathrm{v})$ $\mathrm{CPC}$ at room temperature for $15 \mathrm{~min}$. Finally, $150 \mu \mathrm{L}$ solutions was moved to 96 wells, and the absorbance value was measured at $540 \mathrm{~nm}$ for the calcium quantification analysis. In order to further intuitively observe the effect of composite scaffolds on cell mineralization, PLGA, PDA@PLGA, AuNPs@PDA@PLGA, PLGA/ Lys-g-GO and AuNPs-PDA@PLGA/Lys-g-GO films were prepared and treated under similar conditions to the composite scaffolds. After cells grew on films surface for 21 days, cells were stained with ARS and observed under a microscope (Nikon, E80i, Japan).

\section{Quantitative Real-Time PCR Analysis}

After cells were cultured on different composite scaffolds for 7 days, the osteogenic related gene expression of cells was detected by real-time quantitative PCR (RT-PCR). The total RNA was extracted using TRIzol Reagent (Invitrogen, USA) according to the manufacturer's protocol. Then, the cDNA was synthesized using a PrimeScriptTM RT reagent kit (Takara Bio, Japan) according to the manufacturer's instructions. RT-PCR was performed by Mx3005 (Stratagene, USA) and osteogenesis-related genes including glyceraldehyde-3-phosphate dehydrogenase (GAPDH), type 1 collagen (COL-I), runt-related transcription factor 2 (Runx2) and osteopontin (OPN) were assessed. The primer sequences are shown in Table 1. All experiments were done in triplicate to obtain the average data.

\section{Immunofluorescence Staining}

In order to further detect the effect of different composite scaffolds on cell osteogenic differentiation, films were prepared and treated under similar conditions to the composite scaffolds, and cells were seeded on the surface of films. After 7 days of cell culture, the cells were fixed with paraformaldehyde and rinsed with PBS. Then, the cells were perforated with $0.2 \%(\mathrm{v} / \mathrm{v})$ Triton X-100 (CAS number 9002-93-1) and then incubated with 1\% BSA solution. COL-I (Abcam, ab34710), Runx2 (Abcam, ab114133) and OPN (Abcam, ab63856) primary antibodies were added into the cells and incubated overnight under $4^{\circ} \mathrm{C}$ overnight. After the primary antibody was incubated, FITClabeled secondary antibody was added into cells and 
Table I List of Genes and Primer Nucleotide Sequences

\begin{tabular}{|l|l|l|}
\hline Gene & Forward Primer Sequence & Reverse Primer Sequence \\
\hline OPN & 5-TCAGGACAACAACGGAAAGGG-3' & 5- GGAACTTGCTTGACTATCGATCAC-3' \\
Runx-2 & 5- GCCGGGAATGATGAGAACTA-3' & 5- GGACCGTCCACTGTCACTTT-3' \\
COL-I & 5- CGCTGGCAAGAATGGCGATC-3' & 5- ATGCCTCTGTCACCTTGTTCG-3' \\
GAPDH & 5-TGAACTAACACAGAGGAGGATCAG-3' & 5- GCTTAGGGCATGAGCTTGAC-3' \\
\hline
\end{tabular}

incubated at room temperature for $60 \mathrm{~min}$. The cells were then stained using DAPI for $1 \mathrm{~min}$, and the cells were observed under a fluorescence microscope (Nikon, E80i, Japan).

\section{Repair for Rabbit Radius Defects}

Male New Zealand white rabbits $(2.5 \mathrm{~kg})$ randomly fell into five groups (PLGA, PDA@PLGA, AuNPs-PDA @PLGA, PLGA/Lys-g-GO and AuNPs-PDA@PLGA/ Lys-g-GO, four animals per group) and were used for the radial defect implantation of scaffolds. All experimental animals were provided by Liaoning Changsheng Biotechnology Company, and kept in the Animal Room of Public Health College of Jilin University. In brief, after the experimental animals were completely anesthetized, the forelimb hair of rabbit was removed and the skin was disinfected with iodine. Then, a $3 \mathrm{~cm}$ incision was made in the middle radius on experimental animal, and the radius of about $20 \mathrm{~mm}$ was removed with a chainsaw. Then, the scaffold material was trimmed and the defect site was implanted. Finally, the epidermal wound was sutured and treated with regular disinfection and bandaging. Within 7 days after surgery, penicillin was injected intramuscularly to all experimental animals at a daily dose of 400,000 units, and observed the animals' defecation, wound infection and other conditions every day.

\section{X-Ray and CT Examination}

On postoperative weeks 0,4 and 12 , the effect of different composite scaffolds on bone regeneration was examined with Digital Radio graph (Kodak, CR-400 plus, USA). According to the $\mathrm{X}$-ray results, the new bone regeneration was evaluated by Lane-SandhuX ray score (Table 2). At the same time, 12 weeks after the experiment, the experimental animals were sacrificed and the radial tissue was removed. After the periosseous soft tissue was removed, the radius tissue was fixed with paraformaldehyde and the bone tissue was reconstructed by CT scanner (General Electric, ImatronC300, USA) in 3 D.
Table 2 Lane-Sandhu Radiographic Scoring System

\begin{tabular}{|l|l|}
\hline \multicolumn{2}{|c|}{ Degree of Bone Formation } \\
\hline No new bone formed & 0 \\
The area of new bone accounts for 25\% of the defect area & 1 \\
The area of new bone accounts for 50\% of the defect area & 2 \\
The area of new bone accounts for 75\% of the defect area & 3 \\
The area of new bone accounts for 100\% of the defect area & 4 \\
\hline \multicolumn{2}{|c|}{ Degree of Union } \\
\hline Fracture line is fully visible 0 & 0 \\
Fracture line is partially visible 2 & 2 \\
Fracture line is not visible 4 & 4 \\
\hline \multicolumn{2}{|c|}{ Degree of Medullary Cavity Remodeling } \\
\hline No sign of remodeling & 0 \\
Recanalization of medullary cavity & 2 \\
Cortical bone structure forms after recanalization of medullary & 4 \\
cavity & \\
\hline
\end{tabular}

\section{Histological Evaluation}

On postoperative weeks 12 , bone tissue samples were taken out and fixed with paraformaldehyde. Then, bone samples were completely decalcified in 15\% EDTA solution. After decalcification, samples were embedded in paraffin, and cut into tissue slices. Subsequently, tissue slices were stained with Masson trichrome, H\&E and Sirius Red in accordance with standard protocols, and observed using light microscope (Nikon, E80i, Japan) and polarization microscope (Nikon, Eclipse Ci-L, Japan). The ratio of type I and type III collagen was quantitatively analyzed by Image-pro Plus 6.0 software (Media Cybernetics, USA).

\section{Bio-Safety Assessment}

On postoperative weeks 12 , the experimental animals were sacrificed and a healthy New Zealand white rabbit without surgical treatment was taken as the control group. Important internal organs including heart, liver, spleen and kidney were removed from all experimental animals. Then, a series of slices were cut from the organs tissue and 
stained with H\&E. Finally, the physiological structure of important organs in the experimental animals was observed under a microscope (Nikon, E80i, Japan) to evaluate the biological safety of the composite scaffold.

\section{Statistical Analysis}

All quantitative date were analyzed by one-way analysis of variance using Origin 8.0 software (Origin Lab Corporation, USA). Data are expressed as the mean \pm SD. P value less than 0.05 was considered statistically significant in all analyses.

\section{Results and Discussion \\ Characterization of the GO and Lys-g-GO}

In this study, the GO surface was modified with L-lysine by chemical grafting. We first studied the chemical composition of Lys-g-GO nanoparticles by Fourier infrared spectroscopy (FITR). As shown in Figure 2A, the FITR spectrum of GO exhibited three characteristic peaks at $1052 \mathrm{~cm}^{-1}, 3417 \mathrm{~cm}^{-1}$, and $1730 \mathrm{~cm}^{-1}$, which represented the $\mathrm{C}-\mathrm{O}$ bond, the "O-H stretching" band of hydroxyl groups, and the " $\mathrm{C}=\mathrm{O}$ stretching" band of carboxylic acids (black arrow), respectively. The results indicated the presence of abundant oxygen-containing groups on the GO surface. After grafting L-lysine onto the GO surface, the $\mathrm{C}=\mathrm{O}$ bond representing the amide and carboxylic acid groups changed significantly in the range of 1620 $1630 \mathrm{~cm}^{-1}$ (red arrow). In addition, the stretching peaks representing $\mathrm{OH}$ and $\mathrm{NH}$ also changed significantly at $3420 \mathrm{~cm}^{-1}$ (blue arrow). The results suggested successful grafting of L-lysine onto the GO surface. The TEM observation of the surface morphology of GO and Lys-g-GO revealed that GO presented flake like and wrinkled forms, which are similar to peeled wrinkled flakes, as shown in Figure 2B. Adhered with many substances resembling crystal structure, the surface of Lys-g-GO was rougher than the surface of GO. We speculated that the surface attached amino acids might lead to the formation of this morphology. The above FITR and TEM results proved that L-lysine was successfully grafted onto the GO surface.

\section{Influence of GO and Lys-g-GO on PLGA Materials}

First, we prepared PLGA/Lys-g-GO and PLGA/GO films through blending and studied the effects of Lys-g-GO and GO on the physicochemical properties of PLGA. As shown in Figure $2 \mathrm{C}$, the water contact angle of PLGA is $95.5 \pm 2.61^{\circ}$, the water contact angle of the composites significantly decreased to $85.8 \pm 3.17^{\circ}$ after GO addition. Abundant oxygen-containing functional groups such as hydroxyl, carboxyl and epoxy groups, are present on the surface of GO, which confer GO good hydrophilicity. Compared with PLGA/GO, the surface hydrophilicity of PLGA/Lys-g-GO composites further improved as the contact angle decreased to $79.8 \pm 5.5^{\circ}$. Lysine with strong hydrophilicity can provide hydrophilic groups such as amino groups and carboxyl groups for materials. Grafting lysine onto the GO surface can increase the number of hydrophilic groups of materials to some extent. Figure 2D shows the protein adsorption capacity of each group within one hour. The protein adsorption capacity test with BSA as the model protein demonstrated that the addition of Lysg-GO to PLGA enhanced the protein adsorption capacity of the composite material, which was significantly higher than that in the PLGA/GO group $(\mathrm{P}<0.05)$. We speculated that lysine increases the positive charge of the GO surface in the physiological environment, enhancing the electrostatic interaction between the material and negatively charged BSA. The excellent protein absorption of the material could quickly generate a layer of protein film on the surface to supply more binding sites, improving cell adhesion and migration.

To further study the effect of GO and Lys-g-GO on the biological activity of PLGA materials, we planted MC3T3-E1 cells on different material surfaces and detected the proliferation, adhesion, and osteogenic differentiation of the cells by MTT assay, FITC staining, and PNPP assay, respectively. The results showed that compared with GO, Lys-g-GO had no significant effect on cell proliferation but significantly promote cell adhesion. Analysis of cell images (Figure S1) indicated that the cell growth status and area percentage in the PLGA/Lysg-GO group were better than those in the PLGA/GO group $(\mathrm{P}<0.05)$ after 3 days of growth. Moreover, among the two nanoparticles, Lys-g-GO more obviously improved the osteogenic induction ability of PLGA. The ALP level in the PLGA/Lys-g-GO group was significantly higher than that in the PLGA group, especially on the seventh day $(\mathrm{P}<0.05)$ (Figure S2). Many studies found that lysine could regulate the expression of integrin genes in cells, promoting extracellular matrix formation. ${ }^{44}$ Simultaneously, hydroxylysine is the main component of collagen, which can promote the synthesis and mineralization of collagen in the extracellular matrix. Therefore, 
A

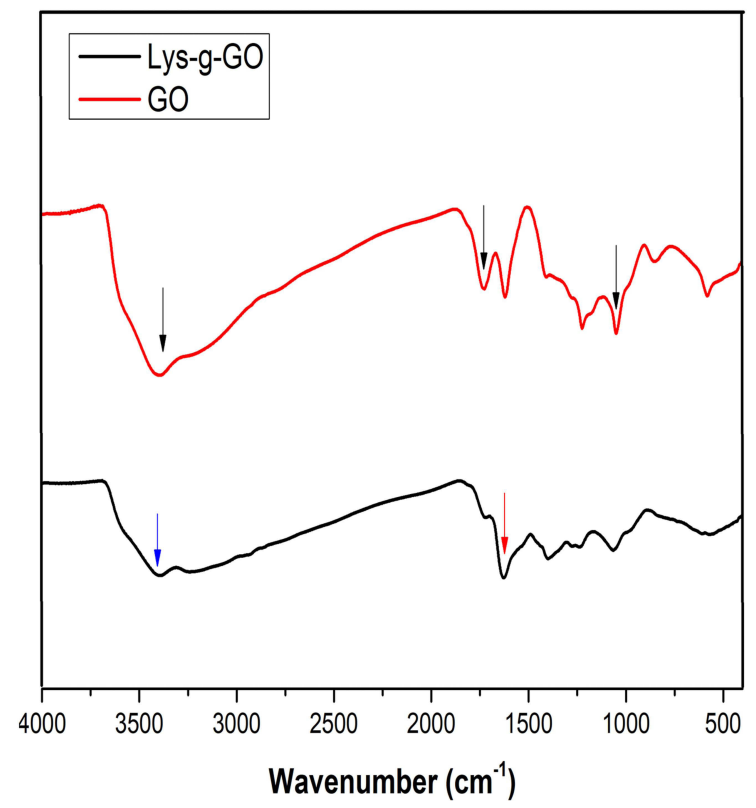

B
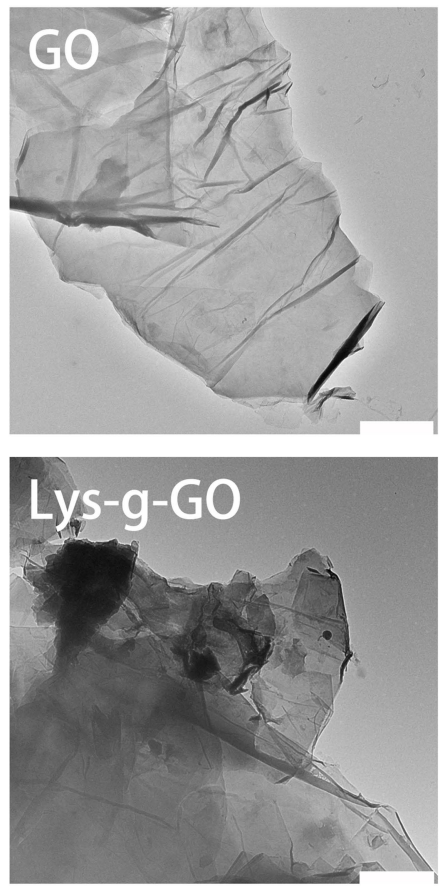

C

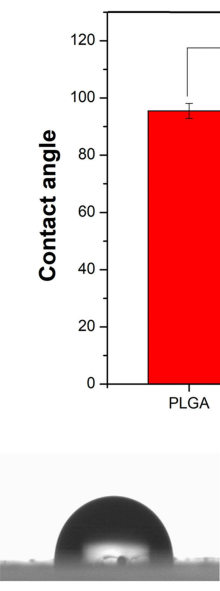

PLGA

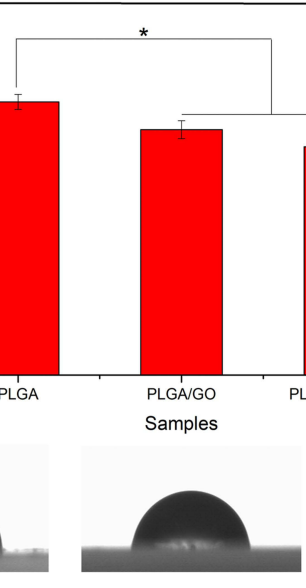

PLGA/GO
D

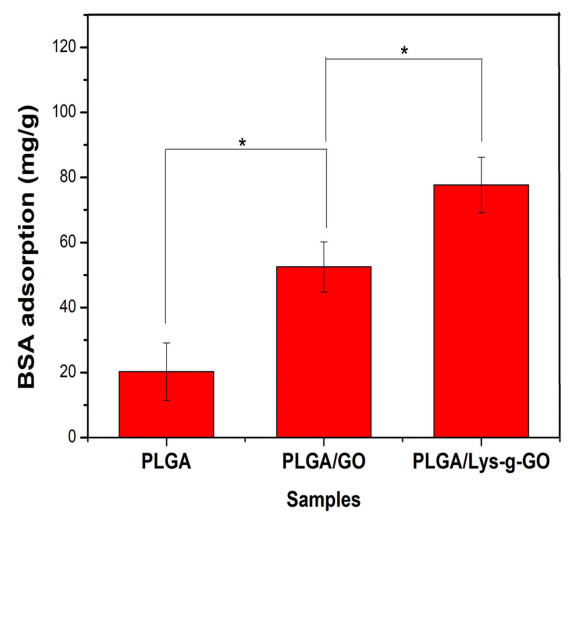

Figure 2 (A) FITR spectra and (B) TEM images of GO and Lys-g-GO nanoparticles, All scale bar are I $\mu$ m. (C) Contact angle analysis of PLGA, PLGA/GO and PLGA/Lysg-GO composite. (D) BSA adsorption efficiency of PLGA, PLGA/GO and PLGA/Lys-g-GO composite, $* \mathrm{P}<0.05, \mathrm{n}=3$.

compared with unmodified GO, Lys-g-GO can further improve the hydrophilicity, protein absorption, and osteogenic induction ability of PLGA.

\section{Characterization of Composite Scaffolds}

Considering the excellent biological activity of Lys-g-GO, we prepared PLGA/Lys-g-GO composite scaffolds by the particle leaching/phase transformation method using PLGA and Lys-g-GO as matrix materials. Additionally, we applied AuNPs-PDA functionalized coatings to the surfaces of the scaffolds by reducing gold ions in the solution to AuNPs with polydopamine. As shown in Figure 3, all scaffolds present very typical through-hole structures with channels interconnecting and running through the whole scaffold. In addition, microchannel structures exist on the macropore surfaces of the scaffolds, the size of which ranges from nanometres to more than ten microns, which can effectively improve nutrient exchange inside the scaffold and show advantages in regulating cell function over the flat pore surface. The pure PLGA scaffold is pure white. After Lys-g-GO addition, the 
A
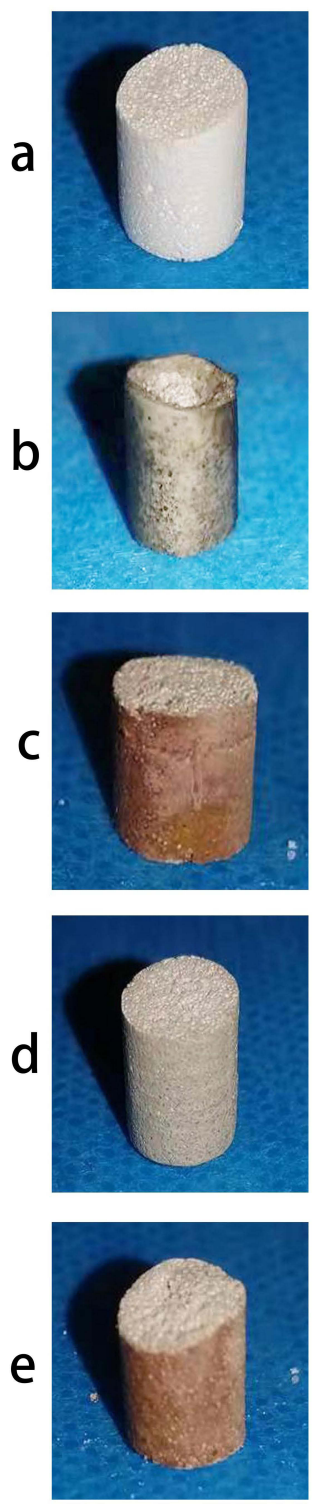

B
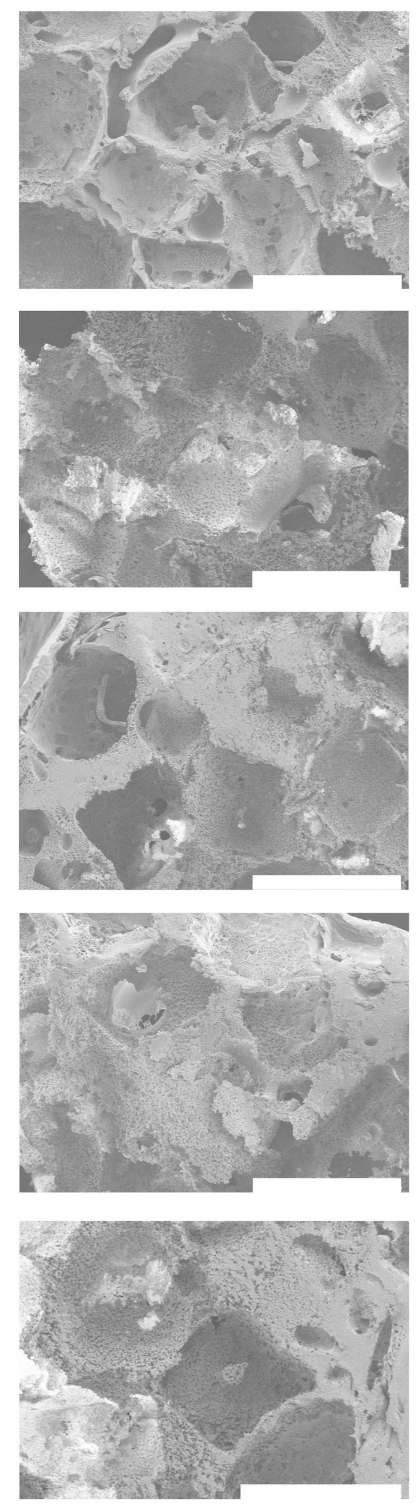

C
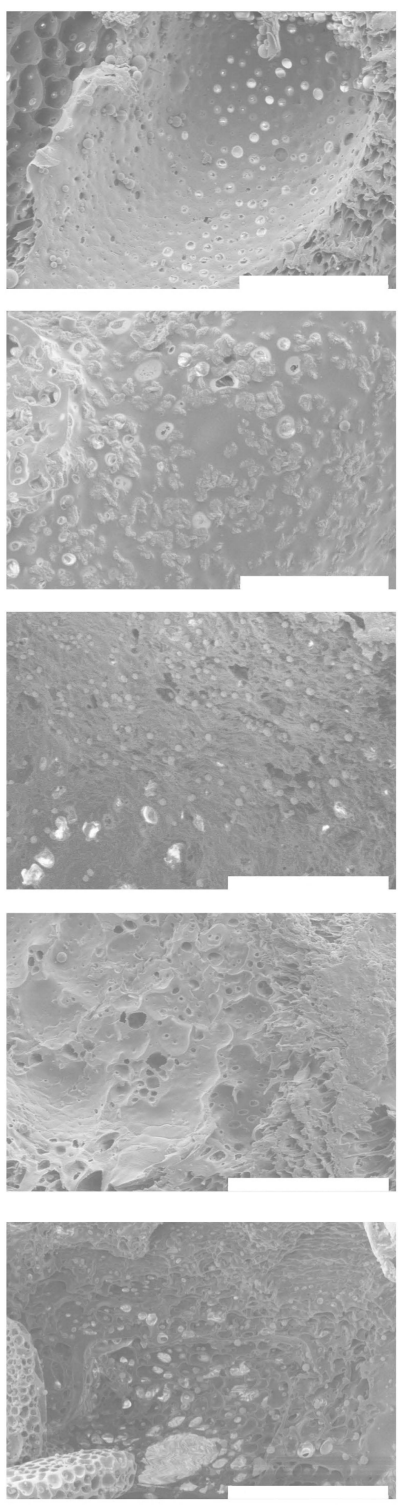

Figure 3 (A) Macroscopic view and (B and C) SEM images of PLGA (a), PDA@PLGA (b), AuNPs-PDA@PLGA (c), PLGA/Lys-g-GO (d) and AuNPs-PDA@PLGA/Lys-g-GO (e) scaffolds. Bar lengths are $400 \mu \mathrm{m}$ (B) and $40 \mu \mathrm{m}$ (C).

general appearance of the scaffold showed no significant change, but the colour was slightly deepened to light grey. After surface modification with polydopamine, the scaffold was further darkened to black, and the whole scaffold appeared dark brown after loading AuNPs. The pore surface of PLGA is relatively flat with scattered microchannel structures. The addition of Lys-g-GO significantly increased the size and number of microchannels on the surface, which may be related to the layered PLGA material caused by the addition of nanoparticles, thus forming more microchannel structures.PDA@PLGA and single PLGA scaffolds had a similar morphology and structure, but the surface of the scaffolds was rougher, and the microchannel diameter reduced, probably due to the polydopamine coating. After loading AuNPs, some agglomerated gold nanoparticles were found on the surface of the scaffold.

We further analysed the surface chemical composition of different scaffolds to confirm functional modification. Figure 4A and B shows the XPS high-resolution scanning spectrum of the scaffold surface with evident peak intensity changes for elements on different scaffold surfaces. The prominent peaks on the surface of the 
A
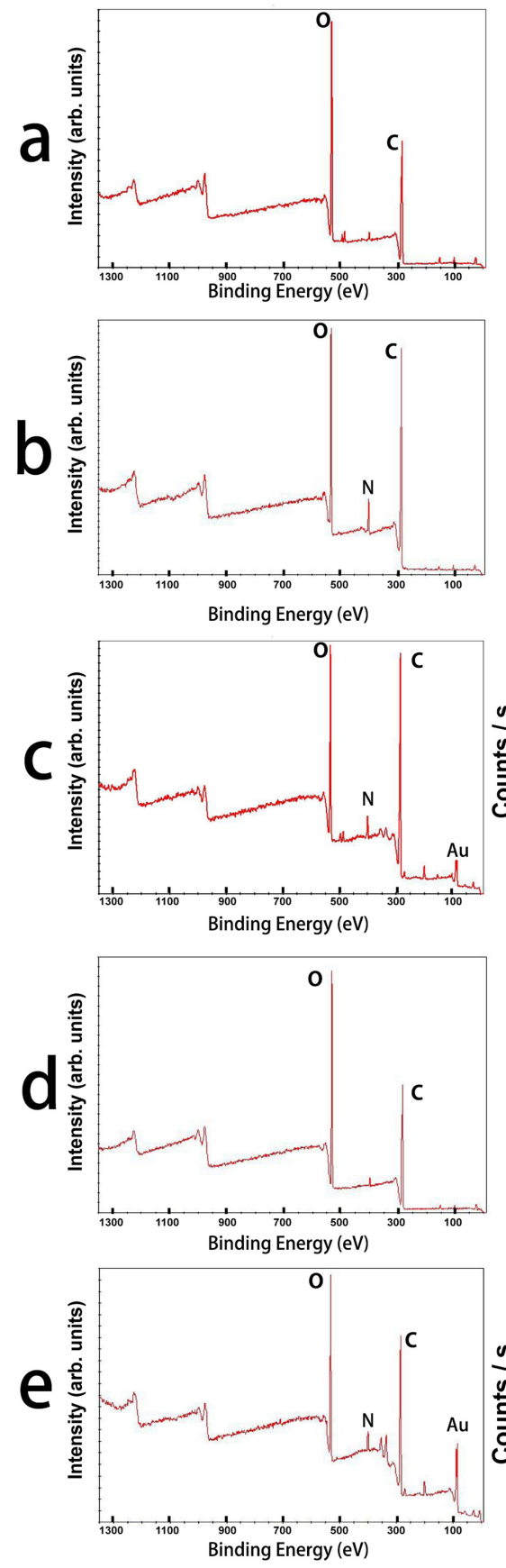

B
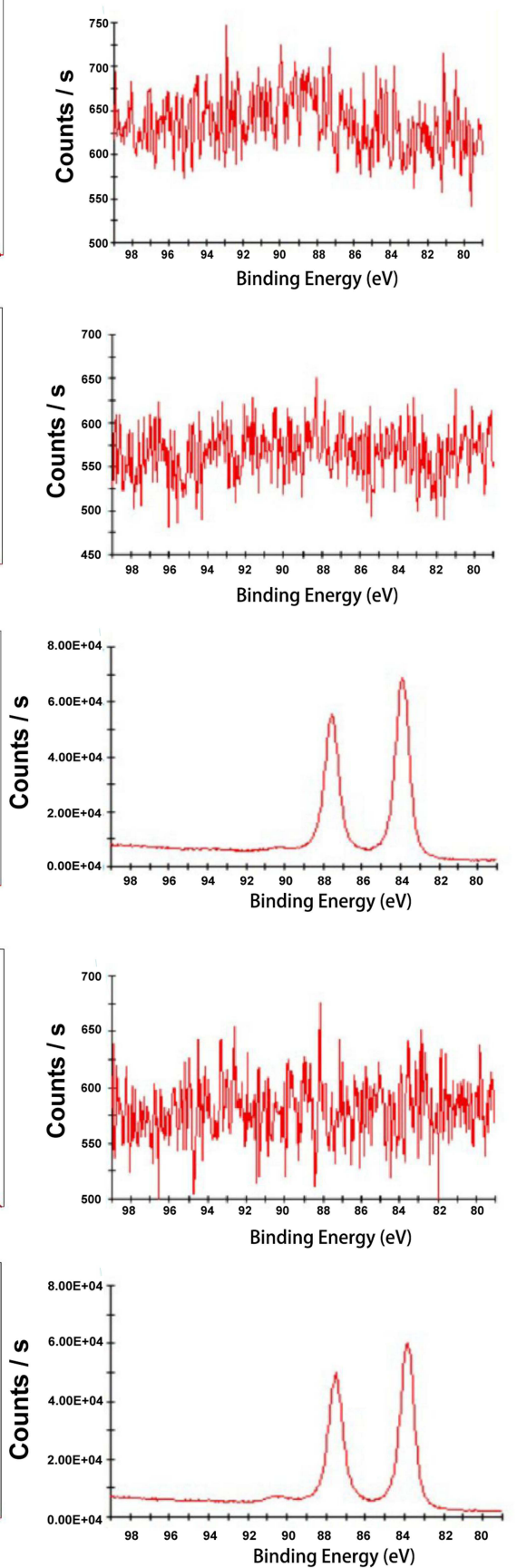

C
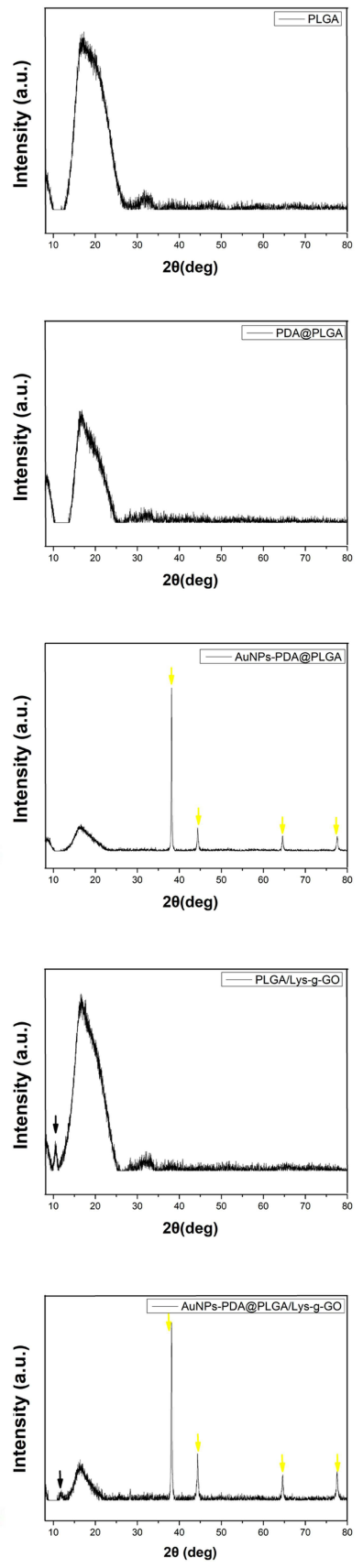

Figure 4 (A) XPS, (C) XRD patterns and (B) the detailed diagram of the Au 4f counts for PLGA (a), PDA@PLGA (b), AuNPs-PDA@PLGA (c), PLGA/Lys-g-GO (d) and AuNPs-PDA@PLGA/Lys-g-GO (e) scaffolds.

PLGA scaffolds were C1S and O1S. After surface modification of PLGA scaffolds with polydopamine, a prominent N1S peak appeared at $400 \mathrm{eV}$, which was caused by the introduction of a surface amine. The elemental composition of the PLGA/Lys-g-GO scaffolds was the same as that of the PLGA scaffolds, and no obvious N1S peak, which may be related to the low Lys-g-GO content. The peak of Au1S was detected after the scaffolds were loaded with AuNPs. The highresolution spectrum of $\mathrm{Au}$ confirmed the presence of zero-valent gold, indicating that AuNPs had deposited on the surface of the scaffolds. We detected the crystal 
structure of different scaffold samples through XRD. Figure 4C shows the XRD patterns of different scaffold samples. We found peaks in the range 13-27 at the $2 \theta$ position of different samples, which was the characteristic peak of PLGA. After modifying scaffolds with polydopamine, the XRD pattern did not change significantly, indicating that polydopamine had no obvious effect on the crystal structure of the scaffolds. After adding Lys-g-GO to the scaffolds, the XRD patterns of the PLGA/Lys-g-GO samples showed a characteristic peak with a corresponding layer spacing of approximately $0.79 \mathrm{~nm}$ at the position of 11 (black arrow), which was a typical characteristic peak of GO, demonstrating the existence of Lys-g-GO in the scaffolds. After AuNPs were loaded on the scaffolds, the characteristic peak of gold appeared at 38, 44, 65, and 78 (yellow arrow). The XPS and XRD results confirmed the completion of scaffold surface modification.

\section{Contact Angle Analysis}

Surface hydrophilicity can significantly affect bone implant material biocompatibility. A hydrophilic surface can effectively promote cell infiltration and adhesion. In this research, we evaluated the wettability of scaffold materials by measuring water droplets contact angles on the surfaces of different materials. As shown in Figure $5 \mathrm{~A}$ and $\mathrm{B}$, the PLGA contact angle decreased significantly after incorporation of Lys-g-GO nanoparticles. After surface modification with polydopamine, the contact angle of the scaffold was further significantly reduced to $29.1 \pm 6.27^{\circ}$, probably due to the rich hydrophilic catechol and nitrogen-containing groups in polydopamine. After the scaffolds were loaded with AuNPs, we found that the contact angle of the scaffold surface increased to a particular level $\left(43.3 \pm 5.06^{\circ}\right)$, which might be due to the hydrophobic AuNPs affecting the binding of the surface elements of the scaffolds with water to some extent. Studies have suggested that the cell adhesion ability significantly improves when the material contact angle is between $0^{\circ}$ and $40^{\circ} .{ }^{45}$ The AuNPs-PDA @PLGA/Lys-g-GO scaffold surface contact angle was 38.7 $\pm 7.85^{\circ}$, which might create a more suitable environment for cell growth.

\section{Mechanical Properties of Composite Scaffolds}

Scaffolds can not only fill the bone defect site but also provide mechanical support when treating bone defects.
Therefore, the mechanical strength of scaffolds is very important for bone repair, especially when applied to weight-bearing sites. We analyzed the compressive strength and elastic modulus of different scaffold materials by mechanical testers. As shown in Figure $5 \mathrm{C}$ and $\underline{\mathrm{D}}$, the compressive strength and elastic modulus of the pure PLGA scaffolds were $1.68 \pm 0.22 \mathrm{MPa}$ and $3.06 \pm 1.02 \mathrm{MPa}$, respectively. After the addition of Lys-g-GO, the compressive strength and elastic modulus of the scaffolds increased to $2.79 \pm 0.15 \mathrm{MPa}$ and $7.44 \pm 2.94 \mathrm{Mpa}$, respectively. The results indicated that Lys-g-GO nanoparticles could effectively improve scaffold mechanical properties. GO can interact with polymers through $\pi-\pi$ interactions, van der Waals forces, and hydrogen bonding, stabilizing the material structure, improving the interface interaction, and effectively enhancing the mechanical strength of materials. ${ }^{46}$ The compressive strength of the PDA@PLGA, AuNPs-PDA@PLGA and AuNPs-PDA @PLGA/Lys-g-GO scaffolds were $1.93 \pm 0.22,1.8 \pm 0.16$, and $2.68 \pm 0.21 \mathrm{MPa}$, respectively. The elastic modulus of the PDA@PLGA, AuNPs-PDA@PLGA and AuNPs-PDA @PLGA/Lys-g-GO scaffolds were $3.1 \pm 1.49,3.9 \pm 2.25$ and $7.2 \pm 1.47 \mathrm{MPa}$, respectively. The compressive strength and elastic modulus of the scaffolds modified with polydopamine or loaded with AuNPs did not change significantly (P $>0.05$ ). The results showed that the AuNPs-PDA functionalized coating did not have a negative effect on the intrinsic mechanical strength of the scaffolds, and the compressive strength of the scaffolds was similar to that of human cancellous bone, which can be an ideal bone replacement material.

\section{Antibacterial Activity of Composite Scaffolds}

An ideal bone implant material should also have certain antibacterial ability, such that it can reduce the use of antibiotics, prevent bacterial infection and improve the success rate of surgery. Recently, many studies have found that GO and AuNPs can improve the antibacterial properties of implant materials to a certain extent. ${ }^{47,48}$ Therefore, in this study, S. aureus and E. coli were used as experimental strains to detect the effects of AuNPsPDA functionalized coatings and Lys-g-GO nanoparticles on the antibacterial properties of PLGA materials. In our previous study, PLGA material was demonstrated to have no obvious antibacterial activity, thus, PLGA was used as the experimental control group. ${ }^{49}$ As shown in Figure 6A, 
A

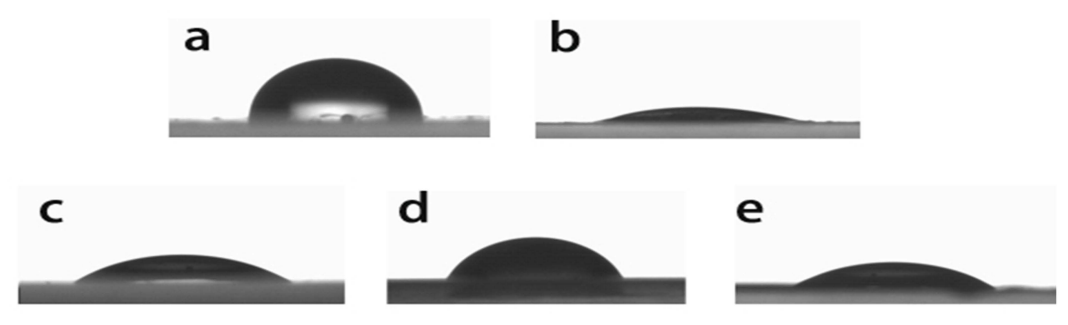

B

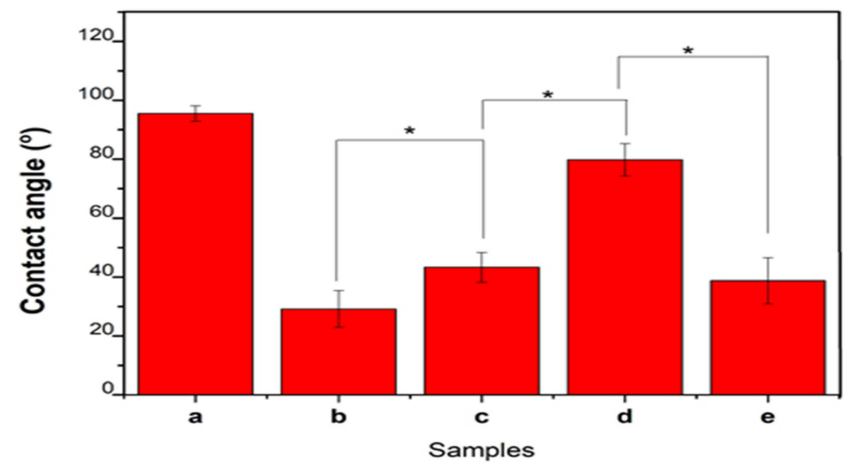

C
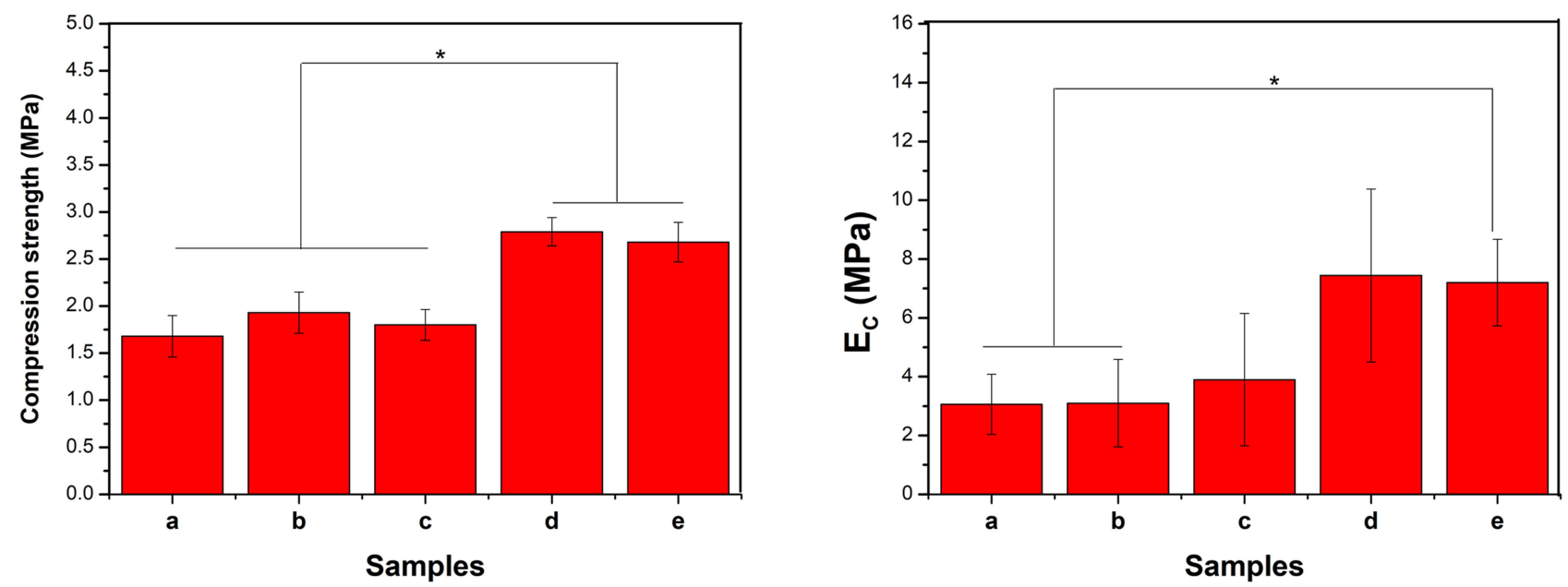

Figure 5 (A and B) Contact angle analysis, (C) Compression strength and (D) Elastic modulus of PLGA (a), PDA@PLGA (b), AuNPs-PDA@PLGA (c), PLGA/Lys-g-GO (d) and AuNPs-PDA@PLGA/Lys-g-GO (e) scaffolds. *P<0.05, error bars represent standard deviation for $n=3$.

the PDA@PLGA group showed a similar OD value as the PLGA group, indicating that the PDA coating had no obvious effect on the antibacterial properties of the material. After loading AuNPs on the surface of the PLGA scaffolds, the OD value of bacterial solutions decreased to a certain extent, but the decreasing trend was not obvious, and a significant difference was found only when scaffold was co-culture with $E$ coli $(\mathrm{P}<0.05)$. Among all scaffolds, the PLGA/Lys-g-GO and AuNPs-PDA@PLGA/Lys-g-GO groups showed the highest bacterial inhibition, and a significant difference was observed compared with the PLGA group $(\mathrm{P}<0.05)$, indicating that Lys-g-GO can enhance the antibacterial activity of PLGA materials. To more intuitively observe the antibacterial properties of different scaffold materials, bacteria were separated from the scaffold sample and plated on LB agar plates, and the 
A
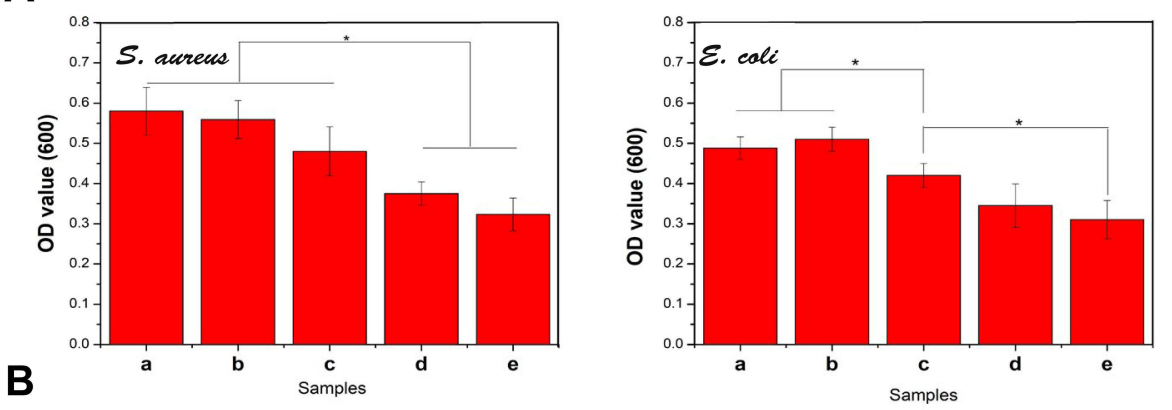

S. aureus

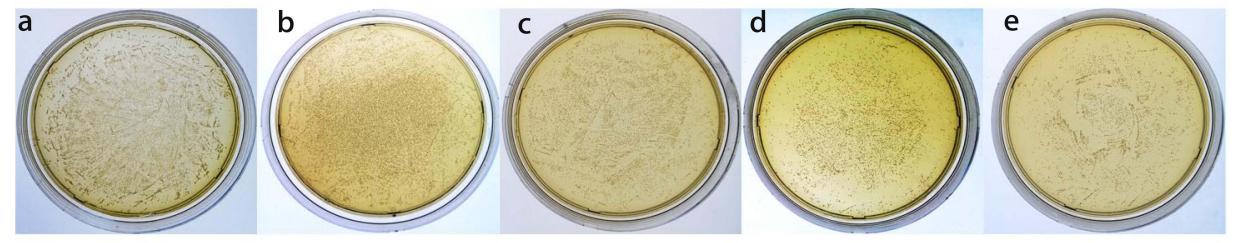

E. cali

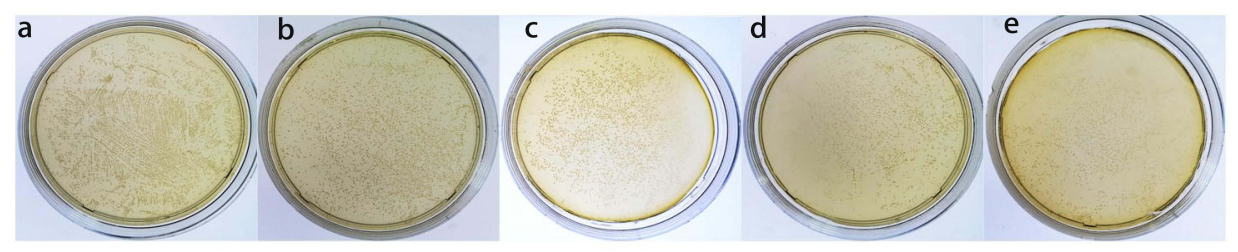

Figure 6 (A) Comparison of the antibacterial efficiency of different composite scaffolds, and (B) Photographs of bacterial colonies formed by E. coli and S. aureus treated with different composite scaffolds. PLGA (a), PDA@PLGA (b), AuNPs-PDA@PLGA (c), PLGA/Lys-g-GO (d) and AuNPs-PDA@PLGA/Lys-g-GO (e).*P<0.05, error bars represent standard deviation for $n=3$.

antibacterial effects of scaffold materials were observed based on the formation of bacterial colonies. As shown in Figure 6B, a large number of colonies were found in the PLGA and PDA@PLGA groups, which almost covered the whole LB culture plate. The AuNPs-PDA@PLGA, PLGA/Lys-g-GO and AuNPs-PDA@PLGA/Lys-g-GO groups performed much better than the PLGA and PDA@PLGA groups in reducing bacterial numbers. Bacterial inhibition was the most obvious in PLGA/Lysg-GO and AuNPs@PLGA/Lys-g-GO groups.

According to the above experimental results, both the AuNPs-PDA functionalized coating and Lys-g-GO can improve the antibacterial activity of PLGA scaffolds to varying degrees. However, compared with Lys-g-GO, AuNPs-PDA coatings have less antimicrobial effects. Some studies have found that free AuNPs can enter the bacterial interior through the cell membrane and destroy the internal structure of the bacteria by inducing oxidative stress. ${ }^{50}$ However, the antimicrobial activity of AuNPs was directly related to the concentration of AuNPs. Some studies have found that AuNPs concentrations above 3.125 and $6.25 \mu \mathrm{g} / \mathrm{mL}$ had inhibitory effects on bacteria. ${ }^{51}$ Therefore, we speculated that the content of AuNPs loaded on the PLGA scaffold surface by PDA coating was low and could not achieve an effective antibacterial concentration. Compared that of the AuNPs-PDA functionalized coating, the antibacterial performance of the scaffold material was improved more obviously by Lys-g-GO. The antibacterial mechanisms of GO materials are mainly divided into two pathways: mechanical damage and induced oxidative stress. First, GO can use its sharp edge structure to cut through the bacterial cell wall, and this mechanical action will destroy the structural integrity of bacteria. ${ }^{52}$ However, this mechanical action mainly occurs in the GO suspension state. When GO is uniformly distributed in a polymer material, this mechanical damage effect is markedly reduced. Another reason for the excellent antibacterial properties of $\mathrm{GO}$ is that $\mathrm{GO}$ can induce oxidative stress reactions in bacteria, thus increasing the pressure on the bacterial cell membrane and destroying the bacterial structure. ${ }^{53}$ We speculated that this characteristic in $\mathrm{GO}$ was the main reason for the improved antibacterial 
properties of the scaffold materials. Furthermore, lysine molecules can increase the number of positive charges of $\mathrm{GO}$, thus, the location of Lys-g-GO on the surface of the scaffold easily captures negatively charged bacteria, and further enhances the antibacterial properties of scaffold materials. More importantly we found that the AuNPsPDA coating and Lys-g-GO had a synergistic effect on improving the antibacterial properties of scaffold materials. Overall, the AuNPs-PDA@PLGA/Lys-g-GO composite scaffold has good antibacterial properties, which can prevent the occurrence of infection and markedly increase the success rate of implantation surgery.

\section{Cell Proliferation and Adhesion}

Changes in the surface properties of scaffolds can interact with cells, affecting cell adhesion, proliferation, differentiation, and other behaviours. The combination of AuNPsPDA coating and Lys-g-GO could significantly change the surface morphology of PLGA scaffolds and improve their hydrophilicity, mechanical strength, and antibacterial properties. We planted MC3T3-E1 cells on the surfaces of different scaffolds and detected cell proliferation to confirm whether these changes in material properties have a positive effect on bone regeneration. Figure $7 \mathrm{~A}$ shows the proliferation of cells on different scaffold surfaces. After 1 day of culture on different scaffolds, no significant change in cell proliferation was found among each group. On the 3rd day, the proliferation rate of cells in the PDA@PLGA group was significantly higher than those in PLGA and PLGA/Lys-g-GO group $(\mathrm{P}<0.05)$, demonstrating that polydopamine modification can effectively improve the cell proliferation. However, a slight decrease in the cell proliferation rate was noted after loading AuNPs on the scaffold surfaces $(\mathrm{P}>0.05)$. After 7 days of cell culture, cell proliferation in each group was similar to that on the 3rd day, but cell proliferation in the AuNPs-PDA@PLGA/Lys-g-GO group improved to a particular extent, and the cell proliferation rate was similar to that in the PDA@PLGA group, and significantly higher than that in PLGA/Lys-g-GO group $(\mathrm{P}<0.05)$.

We created a membrane sheet of scaffold materials in each group and planted MC3T3-E1 cells to further observe the effect of scaffold materials on cell adhesion. After 3 days of cell culture, we observed the adhesion and spread of cells on the material surface. As shown in Figure 7B, although the cells grew well on the surface of pure PLGA materials, the number of cells was significantly lower under a single field of vision, and most of the cells were not fully spread out. After modifying the material surface with polydopamine, the adhered cells exhibited greater numbers, a larger adhered area, and a better spreading state than those in the PLGA and PLGA/Lys-g-GO groups. In the PDA@PLGA, AuNPs-PDA@PLGA and AuNPsPDA@PLGA/Lys-g-GO groups, the cells were almost completely covered material and stacked on top of each other, and the interaction between the cells was more prominent, indicating the strong adhesion behaviour of the cells.

The above results suggested that polydopamine could improve the cell adhesion and proliferation, which may be related to improved hydrophilicity and the abundant catechol-based adhesion groups in polydopamine. ${ }^{54,55}$ However, we found a slight decrease in cell proliferation after loading AuNPs on the scaffold surfaces. Loading of AuNPs, a hydrophobic metal substance, could reduce the material surface hydrophilicity and the exposure of the catechol-based adhesive groups on the scaffold surface to some extent, which may affect the early adhesion and proliferation of the cells in a particular range. In addition, we found the cell proliferation in the AuNPs-PDA @PLGA/Lys-g-GO group was slightly lower in the early stage than that in the PDA@PLGA group, but was significantly improved in the later stage of proliferation. According to the experimental results, Lys-g-GO could also effectively enhance cell proliferation and adhesion behaviour, which might have a particular synergistic effect with the polydopamine coating, overcoming the adverse effects of AuNPs to some extent. The experimental results of cell proliferation and adhesion indicated that the PLGA/ Lys-g-GO scaffolds modified by the AuNPs-PDA functionalized coating can significantly enhance cell adhesion and proliferation.

\section{Cell Mineralization}

Cellular mineralization is a marker of mature bone differentiation, and is widely used to confirm the degree of osteocyte differentiation and the secretion of mineralized extracellular matrix. We evaluated the effects of different scaffold materials on MC3T3-E1 cell mineralization by ARS staining and calcium quantification. As shown in Figure 8A, the PLGA group exhibited the lowest OD value in all groups, demonstrating that the cell calcium deposition in the PLGA scaffolds was the lowest. After modification with polydopamine, the OD value of the PDA@PLGA group was significantly higher than that of the PLGA group $(\mathrm{P}<0.05)$, 
A

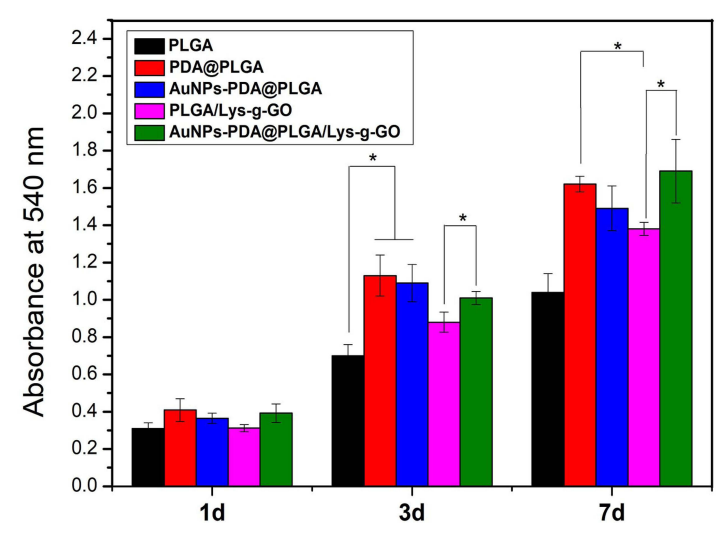

B

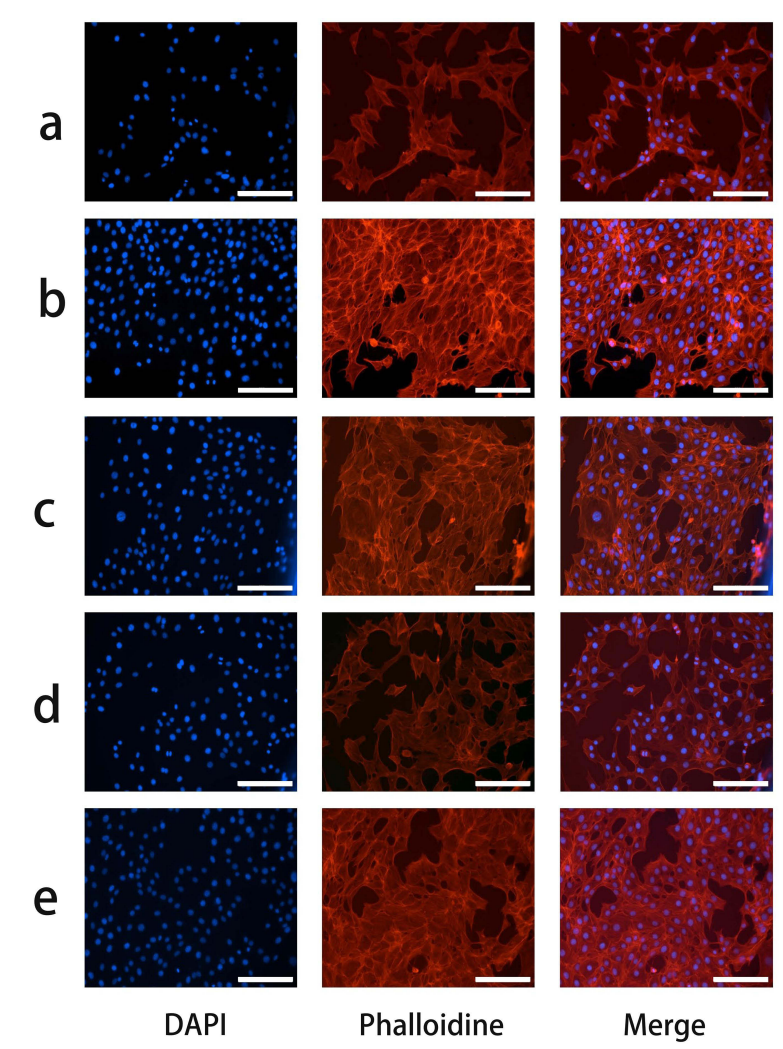

Figure 7 (A) Cell proliferation and (B) morphology of MC3T3-EI cells cultured on PLGA (a), PDA@PLGA (b), AuNPs-PDA@PLGA (c), PLGA/Lys-g-GO (d) and AuNPsPDA@PLGA/Lys-g-GO (e) scaffolds. Scale bar lengths are $200 \mu \mathrm{m}$, *P<0.05, error bars represent standard deviation for $\mathrm{n}=3$.

demonstrating that the calcium deposition content of cells effectively increased. After loading AuNPs on the scaffold surface, the calcium deposition content of cells further increased, and the OD value was close to that in the PLGA/Lys-g-GO group, which was significantly higher than that in the PDA@PLGA group $(\mathrm{P}<0.05)$. Due to the limitation of scaffolds' three-dimensional morphology, we could not directly observe the cell mineralization. Thus, we fabricated different scaffold materials into film sheets and implanted cells. After 21 days of culture, ARS staining was performed for observation. As shown in Figure 8B, the PLGA group had lighter staining and fewer calcium nodules, while the staining intensity and red calcium nodule density in the PDA@PLGA group significantly increased, indicating that polydopamine could promote calcium deposition in the extracellular matrix. Polydopamine can adsorb $\mathrm{Ca}$ and $\mathrm{P}$ ions on the surface of the material through its adhesion ability, thus accelerating the rate of cell mineralization. ${ }^{56}$ After loading AuNPs, the number of 
A

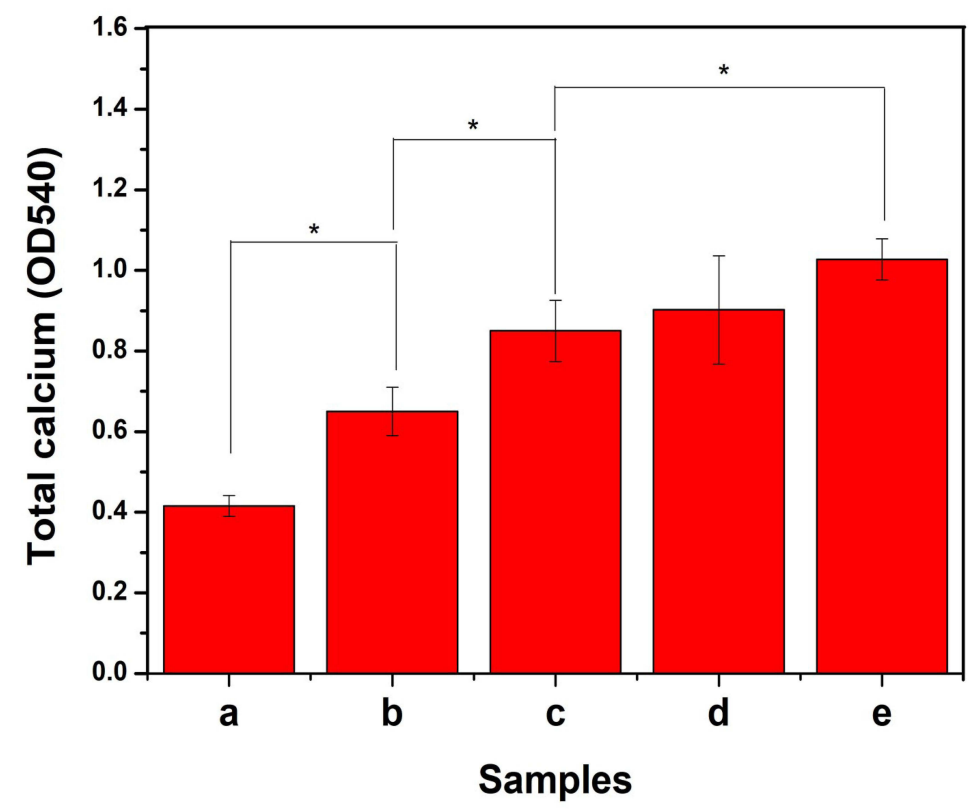

B
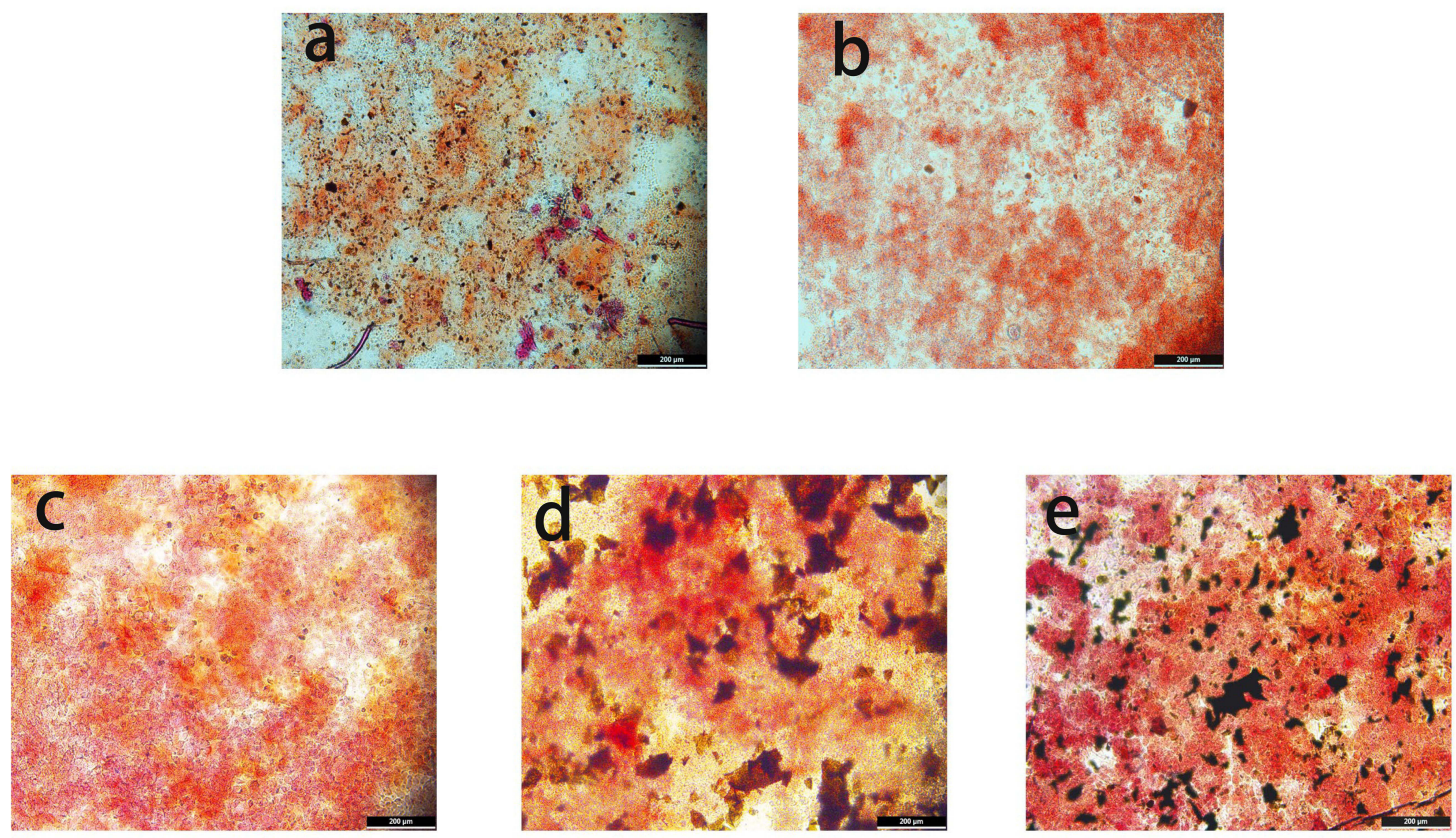

Figure 8 (A) The corresponding quantitative evaluation of calcium content mineral deposition in different composite scaffolds. (B) Alizarin Red staining of MC3T3-EI cells cultured on different films at 2Id. PLGA (a), PDA@PLGA (b), AuNPs-PDA@PLGA (c), PLGA/Lys-g-GO (d) and AuNPs-PDA@PLGA/Lys-g-GO (e). Scale bar lengths are $200 \mu \mathrm{m}, \mathrm{P}<0.05$, error bars represent standard deviation for $\mathrm{n}=3$.

mineralized nodules increased further. Among the sample groups, the AuNPs-PDA@PLGA/Lys-g-GO group exhibited the largest staining area, and red mineralized nodules almost covered the whole field of vision, demonstrating that the AuNPs-PDA functionalized coating and Lys-g-GO presented a synergistic effect in improving cellular calcium deposition.

\section{Bone-Related Gene and Protein Expression}

Bone regeneration involves processes such as cell adhesion, proliferation, osteogenic differentiation and calcium deposition, which are regulated by various genes. For example, the osteogenic transcription factor (Runx-2) is a typical gene expressed early in osteogenic differentiation. ${ }^{57}$ Osteopontin $(\mathrm{OPN})$ is a crucial component of the bone extracellular 
matrix and can be greatly expressed in the middle and late stages of cell osteogenic differentiation. ${ }^{27,58}$ Type I collagen (COL-I) is expressed at the early stage of extracellular matrix secretion, and its expression level gradually increases with cell differentiation and maturation. ${ }^{59}$ Therefore, in this study, the Runx-2, OPN and COL-I genes were selected to detect the bone-related gene expression of cells by RT-PCR. As shown in Figure 9A, after 7 days of cell culture with different scaffolds, the expression level of COL-I in each group increased to some extent compared with the PLGA group. COL-I expression in AuNPs-PDA@PLGA/Lys-g-GO group was significantly higher than that in the PLGA group ( $\mathrm{P}<$ 0.05). Runx-2 expression was not significantly different between the PLGA and PDA@PLGA groups, indicating that polydopamine had no significant effect on inducing

A

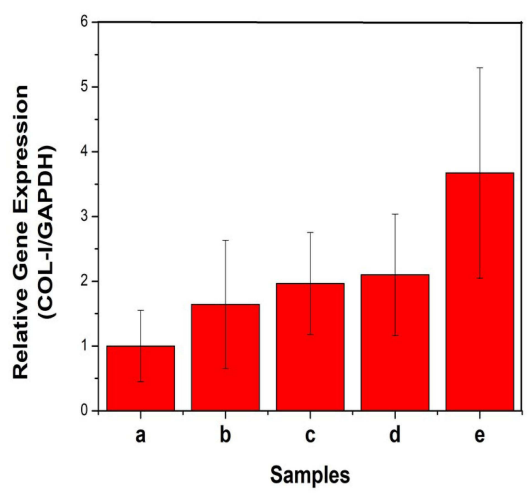

B

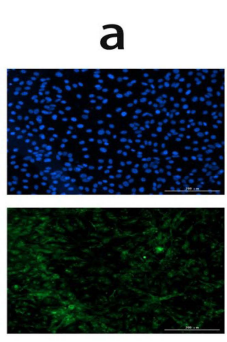

a
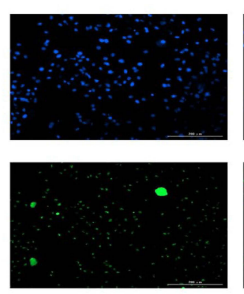

a
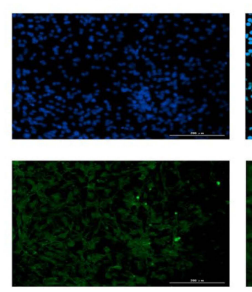

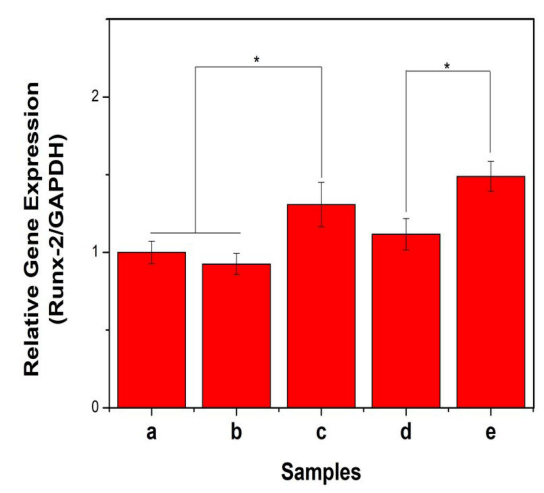

b
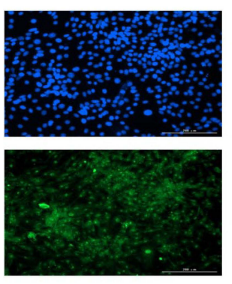

b

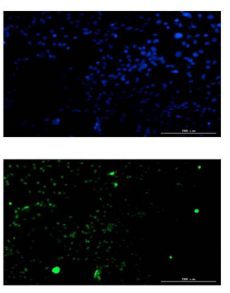

b
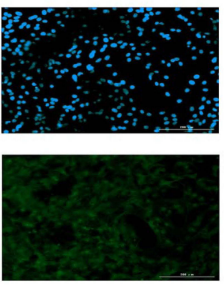

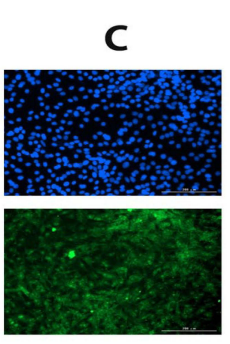

C
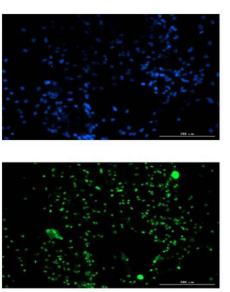

C
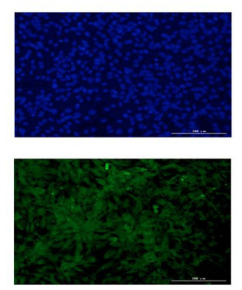

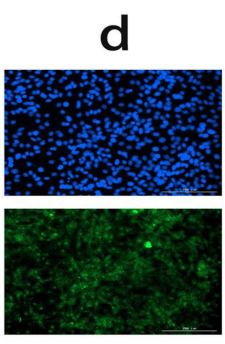

d

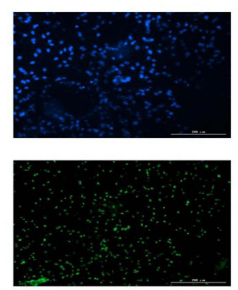

d
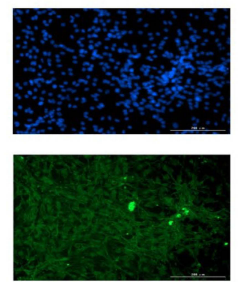

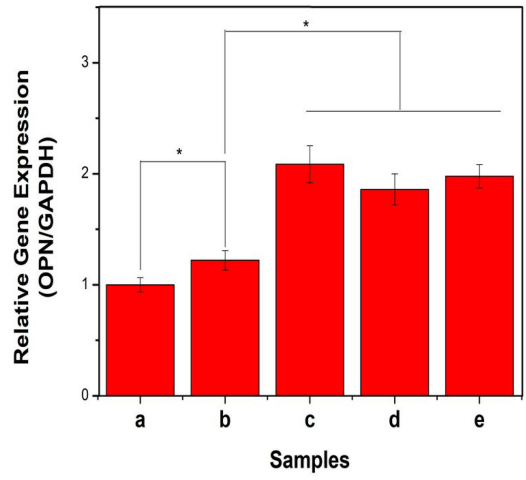

e

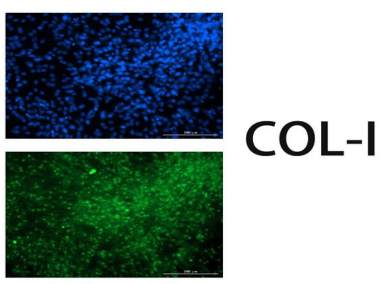

e
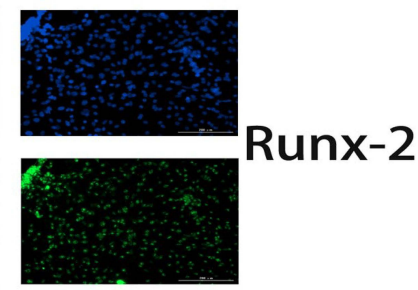

e

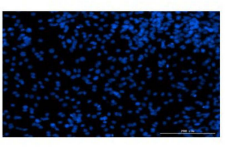

\section{OPN}

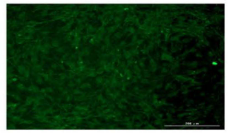

Figure 9 (A) Quantitative real-time PCR analysis and (B) immunofluorescent images for COL-I, Runx-2 and OPN expression by MC3T3-EI cells seeded on PLGA (a), PDA@PLGA (b), AuNPs-PDA@PLGA (c), PLGA/Lys-g-GO (d) and AuNPs-PDA@PLGA/Lys-g-GO (e) composite scaffolds. DAPI staining for nuclei (blue) and FITCconjugated secondary antibody for protein (green). ${ }^{*} \mathrm{P}<0.05$, error bars represent standard deviation for $\mathrm{n}=3$, scale bar lengths are $200 \mu \mathrm{m}$. 
early cell osteogenic differentiation. The expression level of Runx-2 in the AuNPs-PDA@PLGA group significantly increased $(\mathrm{P}<0.05)$ compared with that in the PDA@PLGA and PLGA groups. The Runx-2 level of the cells was further enhanced after modification with AuNPsPDA functionalized coating and Lys-g-GO. Comparing the PLGA/Lys-g-GO group, we found that the Runx-2 level in the AuNPs-PDA@PLGA group was higher, indicating that AuNPs were slightly superior to Lys-g-GO in regulating early osteoblast differentiation. Regarding the expression of OPN, we found that the expression of OPN in the PDA@PLGA group was significantly higher than that in the PLGA group $(\mathrm{P}<0.05)$. After modifying the scaffolds with AuNPs-PDA coating and Lys-g-GO, OPN expression in cells further increased, and the expression level was significantly higher than those in the PDA@PLGA and PLGA group $(\mathrm{P}<0.05)$.

We detected the protein expression of osteogenic factors (COL-I, OPN, and Runx2) by protein immunofluorescence staining to further examine the osteogenic differentiation ability of cells on different scaffold surfaces. As shown in Figure 9B, the PLGA group had the lowest COL-I expression, while a high level of COL-I protein expression appeared in the other groups. After loading with AuNPs, greater Runx2 expression was observed in the fluorescence images of the samples, which was significantly higher than that in the other groups. In terms of OPN protein expression, we found that protein expression in the PDA@PLGA group was higher than that in the PLGA group. After loading AuNPs or the addition of Lys-g-GO, OPN expression was further improved in the samples. In particular, a large area of OPN protein expression appeared in the AuNPs-PDA@PLGA/Lys-g-GO group. The expression analysis of proteins and genes related to osteogenic differentiation showed a high degree of agreement between the two, demonstrating that AuNPs-PDA@PLGA/Lys-g-GO scaffolds could effectively promote cell osteogenic differentiation.

The above experimental results suggested that both the AuNPs-PDA coating and Lys-g-GO could effectively improve the osteogenic induction ability of scaffolds and promote the expression of genes and proteins related to osteogenic differentiation. Many studies demonstrated the strong osteogenic induction ability of $\mathrm{GO}$, and the co-culture of GO and cells could effectively improve the osteogenic differentiation ability of cells, which has been proven again in our research. ${ }^{60,61}$
AuNPs could induce a cellular stress response by entering cells, activating the p38 MAPK signalling pathway to induce the upregulation of genes related to osteoblast differentiation. $^{62}$ In addition, polydopamine improved scaffold hydrophilicity and adhesion, promoted the deposition of calcium and phosphorus ions, and enhanced the osteogenic induction of scaffolds.

\section{In vivo Assessment of Bone Regeneration}

We established rabbit radius defect models and repaired the defect models with different scaffolds to further evaluate the bone repair ability of different scaffold materials. As shown in Figure 10A, we created a $20 \mathrm{~mm}$ defect in the radius of the rabbit and repaired the defect with different scaffold materials. We first observed the healing of the radius by X-ray in different experimental animals. As shown in Figure 10B, neat and clear defects of approximately the same length were found in the radius of all experimental animals at 0 weeks postoperatively; less new bone formation were observed in the PLGA group at 4 weeks, and defect connection and repair were not completed in the following 12 weeks. The results demonstrated the limited bone repair ability of the PLGA scaffold. At 4 weeks, more new bone tissue appeared in the defect sites of the PLGA/Lys-g-GO group and AuNPs-PDA@PLGA/Lys-g-GO group, and the defect sites had achieved preliminary connections compared with the PLGA group. Although large amounts of new bone tissue were also found in the defect site in the AuNPs-PDA@PLGA group, the amount of bone tissue was slightly less than those in the PLGA/Lys-g-GO group and AuNPs-PDA@PLGA/Lys-g-GO group. At 12 weeks, the defect sites of the AuNPs-PDA@PLGA group, PLGA/Lys-g-GO group, and AuNPs-PDA@PLGA/Lysg-GO group were filled with new bone with the convergence of bone edges and the morphology close to normal bone tissue. The defects were not completely filled in the PDA@PLGA group, but the amount of new bone was slightly higher than that in the PLGA group. Then, we conducted Lane-Sandhu X-ray scoring on the bone regeneration of different groups. Figure 10D shows that the AuNPs-PDA@PDA-PLGA/Lys-g-GO group had the highest repair effect score, while the PLGA group had the lowest repair effect score. The repair effect score was as follows: AuNPs-PDA@PLGA/Lys-g-GO (7.25 \pm 2.21$)$ $>$ PLGA/Lys-g-GO (6.75 \pm 2$)>A u N P s-P D A @ P L G A \quad(6$ $\pm 1.41)>$ PDA@PLGA (3.25 \pm 1.25$)>$ PLGA (2.75 \pm 1.5$)$. We took the radius samples from experimental animals 
A
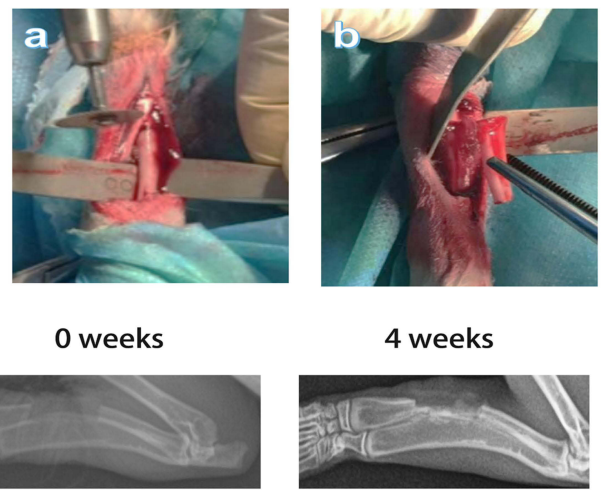

b
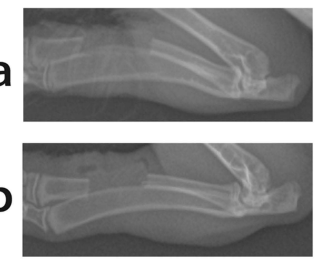

c
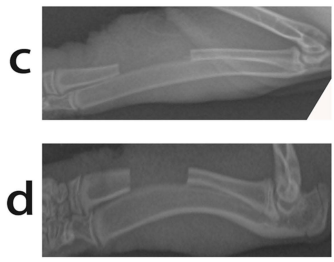

e
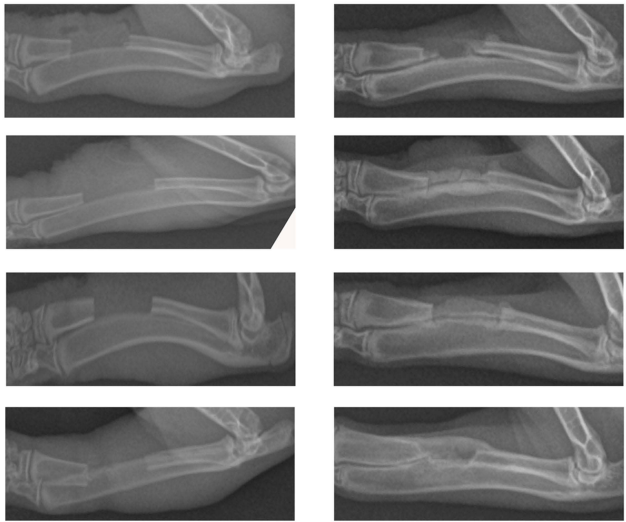

4 weeks

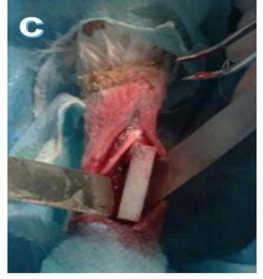

12 weeks
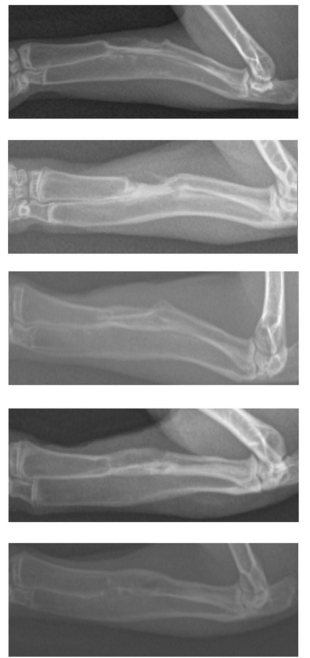

C

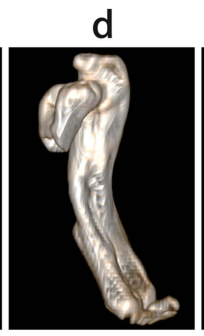

e
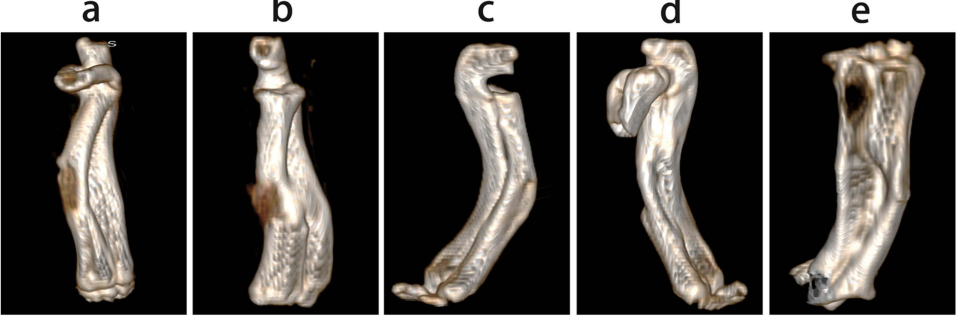

D

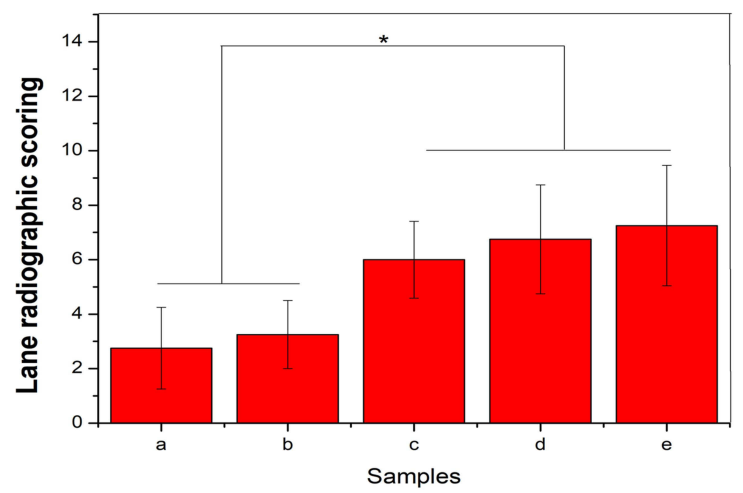

Figure 10 (A) Diagram of animal experiment process, (B) X-ray, (C) CT reconstruction image and (D) Lane-Sandhu radiographic scores, PLGA (a), PDA@PLGA (b), AuNPs-PDA@PLGA (c), PLGA/Lys-g-GO (d) and AuNPs-PDA@PLGA/Lys-g-GO (e) scaffold treatment groups. *P<0.05, error bars represent standard deviation for n=4.

12 weeks postoperatively and conducted threedimensional reconstruction by a CT-scanner, as seen in Figure 10C. The repair effect of the AuNPs-PDA @ PLGA/Lys-g-GO, PLGA/Lys-g-GO, and AuNPs-PDA (a) PLGA groups was more significant, and the radius shape was close to that of normal bone tissue. The bone defect was visible in the radial centre of the PLGA group and PDA@PLGA group. 


\section{Histologic Analysis}

We analysed the bone formation and collagen deposition at the defect site by HE, Masson, and Sirius red staining. As shown in Figure 11A, the scaffold in each experimental group were decomposed into fragments and scattered among tissues. After 12 weeks, the new bone formation was poor in PLGA group and PDA@PLGA group. The tissue of the defect site was mainly hyperplastic connective tissue, and only a small amount of bone tissue was observed. In contrast, the repair effects of the AuNPs-PDA @PLGA/Lys-g-GO group, PLGA/Lys-g-GO group, and AuNPs-PDA@PLGA group were significantly better than those of other two groups. The amount of new bone at the bone defects was larger with many formations of lamellar bone, suggesting the excellent repair effect of the implant. Figure 11B shows the Masson staining results, a small number of blue collagen fibre was found in the PLGA group. In contrast, we found more blue collagen fibre in

A
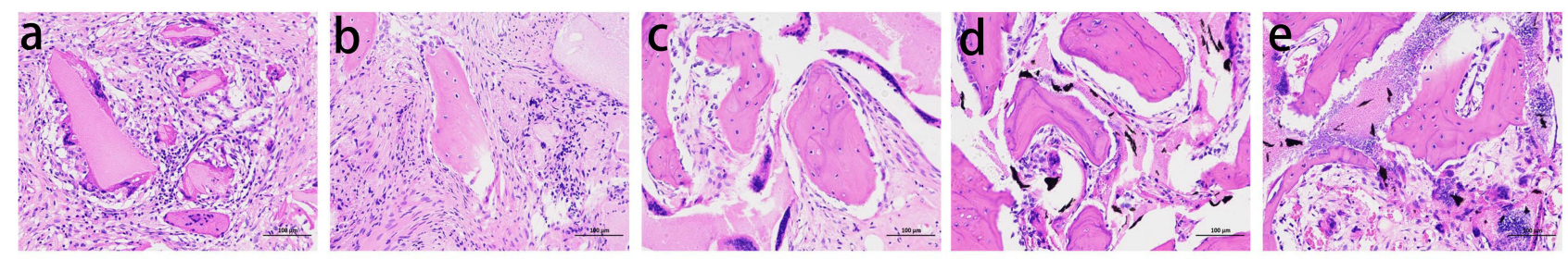

B
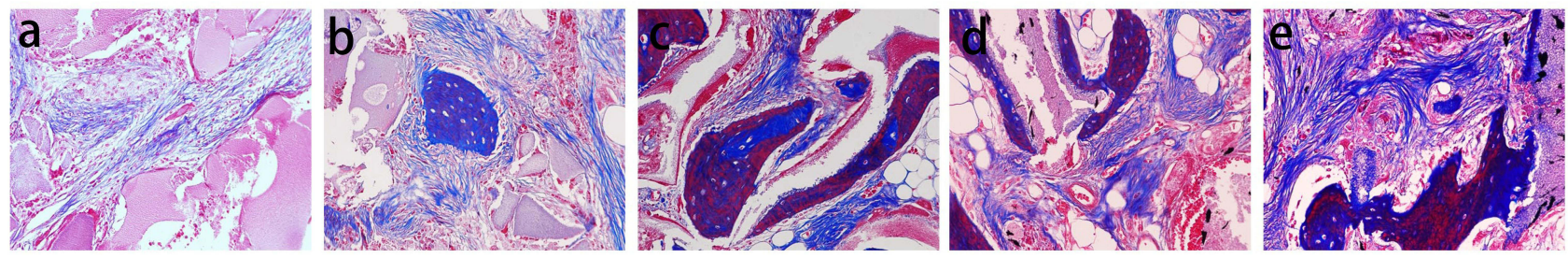

\section{C}
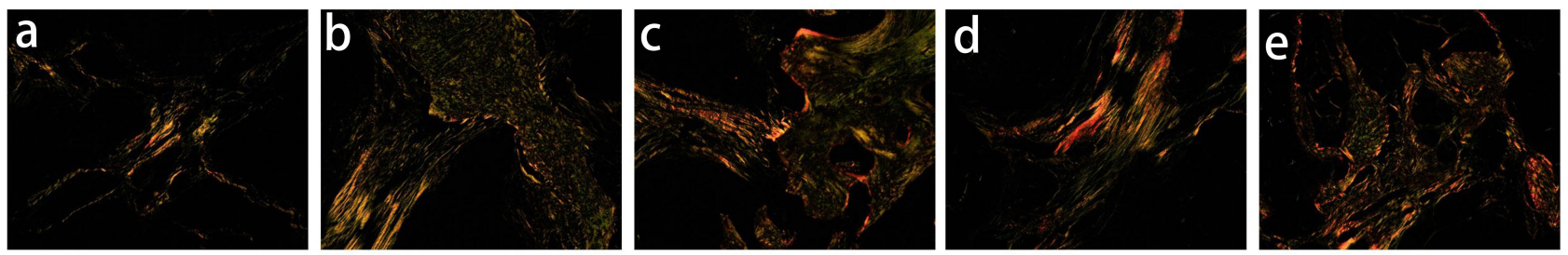

D

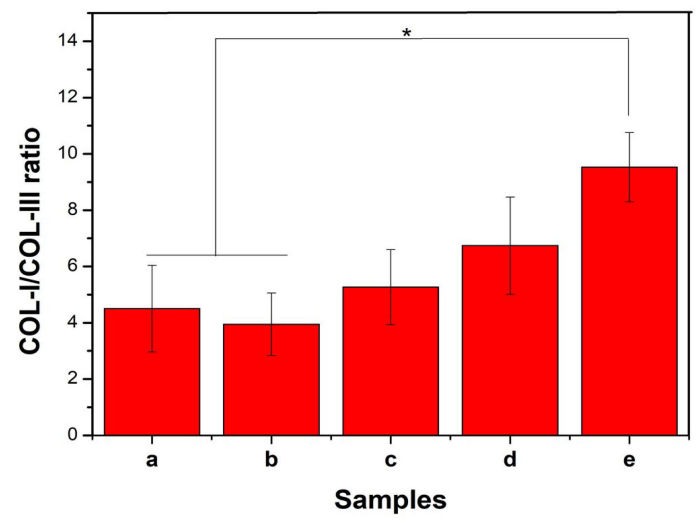

Figure II Histological analysis using H\&E (A), Masson trichrome (B), Sirius red (C) staining and the COL-I/COL-III area ratio (D). PLGA (a), PDA@PLGA (b), AuNPs-PDA @PLGA (c), PLGA/Lys-g-GO (d) and AuNPs-PDA@PLGA/Lys-g-GO (e). Magnification, 200x. 
the PDA@PLGA group, indicating that surface modification with polydopamine could promote collagen secretion in the bone defect sites. More mature collagen fibre appeared in the PLGA/Ly-g-GO and AuNPs-PDA (a) PLGA groups, accompanied by an osteoid matrix. Moreover, we found that the AuNPs-PDA@PLGA/Lysg-GO group contained the largest collagen fibre area, and blue collagen fibres covered most of the defective tissues.

In the organic components of normal bone tissue, the COL-I content is more than $90 \%$. COL-I can provide binding sites for bone tissue biomineralization and promote cell differentiation, determining the performance of new bone to a large extent. Therefore, COL-I can evaluate the maturation of new bone to a certain extent. This research employed Sirius red staining to evaluate the collagen composition in sample tissues. As shown in Figure $11 \mathrm{C}$, the amount of COL-I in all groups was higher than that of COL-III, indicating that the defect had passed the initial stage of fracture repair. However, the red/yellow region was larger in the AuNPs-PDA@PLGA/Lys-g-GO group and PLGA/Lys-g-GO group, and the red/yellow region was smaller while the green region was larger in PLGA group and PDA@PLGA group. As shown in Figure 11D, the COL-I/COL-III ratio of the PLGA, PDA@PLGA, AuNPs-PDA@PLGA, PLGA/Lys-g-GO and AuNPs-PDA@PLGA/Lys-g-GO group were approximately $4.5 \pm 1.54,3.95 \pm 1.11,5.27 \pm 1.33,6.74 \pm 1.73$ and $9.53 \pm 1.23$, respectively. Analysis of the proportion of COL-I and COL-III demonstrated a significantly higher proportion of COL-I/COL-III in the AuNPs-PDA (a) PLG/Lys-g-GO group than that in the PLGA and PDA@PLGA groups $(\mathrm{P}<0.05)$, suggesting that the collagen content of the new tissue was close to that of the normal bone tissue. The histological test results indicated that the implant of AuNPs-PDA@PLGA/Lys-g-GO scaffold could achieve a good repair effect on bone defects, and the new tissue composition was close to that of the normal bone tissue.

\section{Bio-Safety Assessment of Composite Scaffolds}

Imaging and histological experiments confirmed that the Lys-g-GO and AuNPs-PDA functionalized coatings could significantly improve the bone repair ability of PLGA scaffolds. However, such nanomaterials could enter other parts of the body through body fluid circulation. Therefore, the bio-safety of scaffold materials also needs to be studied. At 12 weeks postoperatively, we conducted histopathological observation of the heart, liver, spleen, and kidney of rabbits. As shown in Figure 12, in the control group, HE results for heart tissue sections showed normal arrangement of myocardial fibre tissue, no significant changes in myocardial interstitium, and no granular degeneration, steatosis or other changes in myocardial cells. The results for liver HE stained sections showed that the structure of hepatic lobules was intact, hepatocytes were orderly arranged, and hepatocytes showed no degeneration or sclerosis. HE results for spleen tissue sections showed that the morphology of red pulp and white pulp was normal, and no significant increase of endothelial cells and polynucleated white blood cells was evident in the sinus. HE results showed a normal size and number of glomeruli, and there was no obvious infiltration of inflammatory cells in the renal interstitium. Compared with the normal rabbit viscera of the control group, no abnormal cell or structural changes were observed in the viscera of animals in all experimental groups, suggesting that the scaffold materials in each group did not cause significant damage to the visceral organs. The above experimental results indicated the excellent biocompatibility and bone repair ability of the AuNPs-PDA@PLGA/Lys-g-GO composite porous scaffold prepared by us.

The treatment of large bone defects has always been the focus of orthopaedic research. Although therapeutic modalities such as autologous bone grafts are effective for bone regeneration, many drawbacks have limited their development, such as immune reactions, limited donor sources and transfer of pathogens. With the continuous development of tissue engineering technology, an increasing number of researchers have paid attention to the use of synthetic degradable polymers to solve the drawbacks existing in the traditional treatment of bone defects and promote the repair of bone defects. The ideal bone repair material should have good hydrophilicity, mechanical strength, antibacterial properties, and osteogenic induction ability, provide an appropriate environment for cell growth and proliferation, and promote the healing rate of bone tissue. This research employed Lysg-GO nanoparticles and AuNPs-PDA coatings to optimize the physical and chemical properties of PLGA scaffolds and improve their tissue repair ability. The results showed that combining the AuNPs-PDA functionalized coating and Lys-g-GO nanoparticles could significantly improve the hydrophilicity, mechanical strength, and antibacterial properties of the PLGA 


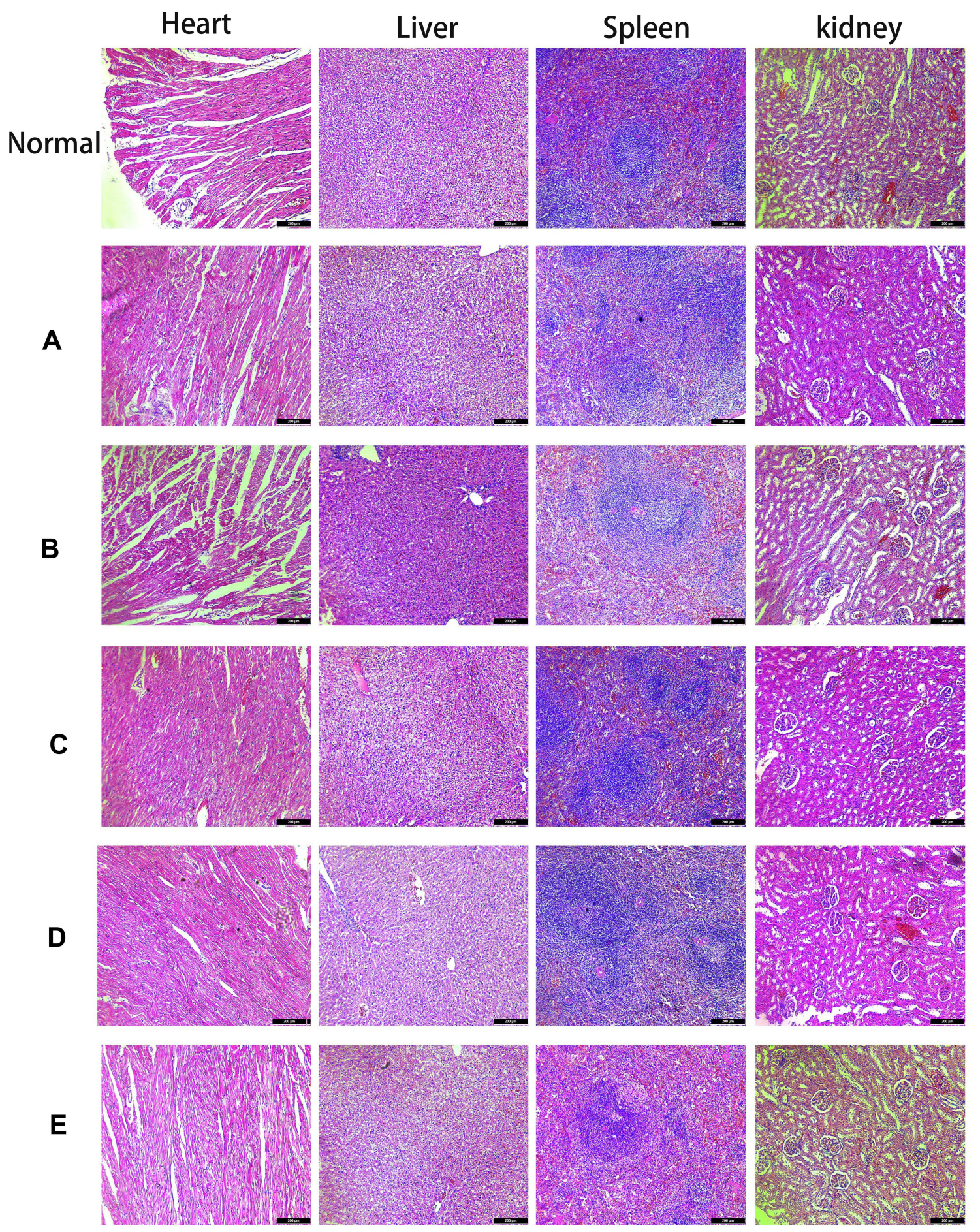

Figure 12 At 12 weeks after surgery, H\&E staining sections of important organs (heart, liver, spleen and kidney) in experimental animals. PLGA (A), PDA@PLGA (B), AuNPs-PDA@PLGA (C), PLGA/Lys-g-GO (D) and AuNPs-PDA@PLGA/Lys-g-GO (E). Scale bar lengths are $200 \mu \mathrm{m}$.

scaffold. Lys-g-GO and AuNPs-PDA coatings exhibited synergistic effects on promoting cell proliferation, adhesion, osteogenic differentiation, and calcium deposition. The animal experiment results showed that AuNPs-PDA @PLGA/Lys-g-GO composite porous scaffold presented an excellent bone repair ability, and the new bone formation and collagen deposition at the defect sites were effectively improved. Additionally, scaffold materials had good biocompatibility and had no obvious toxicity or side effects on the important organs (liver, spleen, kidney and heart) of rabbits. Therefore, we believe that the scaffold materials that we prepared have good clinical 
development prospects and may play an essential role in bone tissue regeneration medicine.

\section{Conclusions}

In this study, a AuNPs-PDA coating modified PLGA/ Lys-g-GO composite scaffold with a loosened skeleton was fabricated. The scaffold has a channel-like pore structure and full 3D interconnection, and the surface of pores has a honeycomb-like structure composed of microvoids, which was suitable for cell infiltration and nutrient transport. The application of Lys-g-GO nanoparticles and AuNPs-PDA coating markedly enhanced the hydrophilicity, mechanical properties and antibacterial properties of the PLGA scaffold, resulting in good osteogenic activity. In vivo results demonstrated that the AuNPs-PDA@PLGA/Lys-g-GO composite scaffolds were effective in repairing rabbits radial defect. Successful regeneration of bone tissue was confirmed by increased mineralized collagen deposition and bone content. This study demonstrated that simultaneously using Lys-g-GO nanoparticles and AuNPs-PDA coating for functional modification of bone implants is a promising strategy, and the AuNPs-PDA@PLGA/Lysg-GO composite scaffold shows promise use in clinical applications for the treatment of bone defects.

\section{Data Sharing Statement}

The raw data used and/or analyzed during the current study are available from the corresponding author on reasonable request.

\section{Ethics Approval and Consent to Participate}

Animals use and care were approved with the Institutional Animal Care and Use Committee of Jilin University, China (Ethical Approval No. 2019132) and were performed in accordance with the university's guidelines. Furthermore, all animal experiments comply with the National Institutes of Health guide for the care and use of laboratory animals (NIH Publications No. 8023, revised 1978).

\section{Acknowledgments}

This work was supported by the National Major Program of New Drug Research and Development; Jilin Provincial Science and Technology Agency Foundation, Key Research and Development Project (20200404137YY); Jilin Province Educational Science Research Project
(JJKH20211168KJ); Jilin Provincial Development and Reform Commission Project (2019C051-6); Specialized Direct Subordinated Program from Finance Department of Jilin Province (Grant No. 2020SCZT065).

\section{Disclosure}

There are no conflicts of interest to declare.

\section{References}

1. Wang J, Li D, Li T, et al. Gelatin tight-coated poly(lactide-co-glycolide) Scaffold Incorporating rhBMP-2 for bone tissue engineering. Materials. 2015;8(3):1009-1026. doi:10.3390/ma8031009

2. Ruan J, Wang X, Yu Z, et al. Enhanced physiochemical and mechanical performance of Chitosan-grafted graphene oxide for superior osteoinductivity. Adv Funct Mater. 2016;26(7):1085-1097. doi:10. 1002/adfm.201504141

3. Chan KH, Lee WH, Zhuo SM, Ni M. Harnessing supramolecular peptide nanotechnology in biomedical applications. Int $J$ Nanomedicine. 2017;12:1171-1182. doi:10.2147/ijn.s126154

4. Ma HB, Su W, Tai Z, et al. Preparation and cytocompatibility of polylactic acid/hydroxyapatite/graphene oxide nanocomposite fibrous membrane. Chinese Sci Bull. 2012;57(23):3051-3058. doi:10.1007/ s11434-012-5336-3

5. Takeoka Y, Hayashi M, Sugiyama N, Yoshizawa-Fujita M, Aizawa M, Rikukawa M. In situ preparation of poly(L-lactic acid-coglycolic acid)/hydroxyapatite composites as artificial bone materials. Polymer J. 2015;47(2):164-170. doi:10.1038/pj.2014.121

6. Gao TL, Cui W, Wang Z, et al. Photo-immobilization of bone morphogenic protein 2 on PLGA/HA nanocomposites to enhance the osteogenesis of adipose-derived stem cells. RSC Adv. 2016;6 (24):20202-20210. doi:10.1039/c5ra27914c

7. Curran JM, Fawcett S, Hamilton L, et al. The osteogenic response of mesenchymal stem cells to an injectable PLGA bone regeneration system. Biomaterials. 2013;34(37):9352-9364. doi:10.1016/j.biomaterials.2013. 08.044

8. Kim SE, Yun Y-P, Shim K-S, Park K, Choi S-W, Suh DH. Effect of lactoferrin-impregnated porous poly(lactide-co-glycolide) (PLGA) microspheres on osteogenic differentiation of rabbit adipose-derived stem cells (rADSCs). Coll Surf B Biointerfaces. 2014;122:457-464. doi:10.1016/j.colsurfb.2014.06.057

9. Sun X, Cheng L, Zhao J, et al. bFGF-grafted electrospun fibrous scaffolds via poly(dopamine) for skin wound healing. J Mater Chemi B. 2014;2(23):3636-3645. doi:10.1039/c3tb21814g

10. Fu C, Bai H, Hu Q, Gao T, Bai Y. Enhanced proliferation and osteogenic differentiation of MC3T3-E1 pre-osteoblasts on graphene oxide-impregnated PLGA-gelatin nanocomposite fibrous membranes. RSC Adv. 2017;7(15):8886-8897. doi:10.1039/c6ra26020a

11. Chan KH, Zhuo SM, Ni M. Priming the surface of orthopedic implants for osteoblast attachment in bone tissue engineering. Int J Med Sci. 2015;12(9):701-707. doi:10.7150/ijms. 12658

12. Shin YC, Lee JH, Jin L, et al. Stimulated myoblast differentiation on graphene oxide-impregnated PLGA-collagen hybrid fibre matrices. J Nanobiotechnology. 2015;13(1):11. doi:10.1186/s12951-015-0081-9

13. Kim S, Park M, Jeon O, Choi C, Kim B. Poly(lactide-co-glycolide) /hydroxyapatite composite scaffolds for bone tissue engineering. Biomaterials. 2006;27(8):1399-1409. doi:10.1016/j.biomaterials.20 05.08.016

14. Armentano I, Dottori M, Puglia D, Kenny JM. Effects of carbon nanotubes (CNTs) on the processing and in-vitro degradation of poly (DL-lactide-co-glycolide)/CNT films. J Mater Sci. 2008;19(6):23 77-2387. doi:10.1007/s10856-007-3276-2 
15. Jin L, Lee JH, Jin OS, et al. Stimulated osteogenic differentiation of human mesenchymal stem cells by reduced graphene oxide. J Nanosci Nanotechnol. 2015;15(10):7966-7970. doi:10.1166/jnn. 2015.11223

16. Rozova EY, Zoolshoev ZF, Kuryndin IS, Saprykina NN, Elyashevich GK. Sorption and mechanical properties of Chitosan/ graphene oxide composite systems. Russian J Appl Chem. 2019;92 (3):415-422. doi:10.1134/s1070427219030121

17. Mohammadi S, Shafiei SS, Asadi-Eydivand M, Ardeshir M, SolatiHashjin M. Graphene oxide-enriched poly(epsilon-caprolactone) electrospun nanocomposite scaffold for bone tissue engineering applications. J Bioact Compat Polym. 2017;32(3):325-342. doi:10. $1177 / 0883911516668666$

18. Zhang $\mathrm{K}$, Zheng $\mathrm{H}$, Liang S, Gao C. Aligned PLLA nanofibrous scaffolds coated with graphene oxide for promoting neural cell growth. Acta Biomaterialia. 2016;37:131-142. doi:10.1016/j.actbio. 2016.04.008

19. Shin YC, Lee JH, Jin L, et al. Stimulated myoblast differentiation on graphene oxide-impregnated PLGA-collagen hybrid fibre matrices. J Nanobiotechnol. 2015;13(1). doi:10.1186/s12951-015-0081-9

20. Dalgic AD, Alshemary AZ, Tezcaner A, Keskin D, Evis Z. Silicatedoped nano-hydroxyapatite/graphene oxide composite reinforced fibrous scaffolds for bone tissue engineering. J Biomater Appl. 2018;32(10):1392-1405. doi:10.1177/0885328218763665

21. Crowder SW, Prasai D, Rath R, Balikov DA, Bae H, Bolotin KI, et al. Three-dimensional graphene foams promote osteogenic differentiation of human mesenchymal stem cells. Nanoscale. 2013;5 (10):4171-4176. doi:10.1039/c3nr00803g

22. Liu YX, Fang N, Liu B, Song LN, Wen BY, Yang DZ. Aligned porous chitosan/graphene oxide scaffold for bone tissue engineering. Mater Lett. 2018;233:78-81. doi:10.1016/j.matlet.2018.08.108

23. Ren L, Pan S, Li H, et al. Effects of aspirin-loaded graphene oxide coating of a titanium surface on proliferation and osteogenic differentiation of MC3T3-E1 cells. Sci Rep. 2018;8(1):15143. doi:10.1038/ s41598-018-33353-7

24. La W-G, Jung M-J, Yoon J-K, et al. Bone morphogenetic protein-2 for bone regeneration - dose reduction through graphene oxide-based delivery. Carbon. 2014;78:428-438. doi:10.1016/j.carbon.2014.07.023

25. Singh V, Kumar V, Kashyap S, et al. Graphene oxide synergistically enhances antibiotic efficacy in vancomycin-resistant Staphylococcus aureus. Acs Appl Bio Mater. 2019;2(3):1148-1157. doi:10.1021/ acsabm.8b00757

26. Yang Z, Si J, Cui Z, et al. Biomimetic composite scaffolds based on surface modification of polydopamine on electrospun poly(lactic acid)/cellulose nanofibrils. Carbohydr Polym. 2017;174:750-759. doi:10.1016/j.carbpol.2017.07.010

27. Wu J, Li L, Fu C, et al. Micro-porous polyetheretherketone implants decorated with BMP-2 via phosphorylated gelatin coating for enhancing cell adhesion and osteogenic differentiation. Coll Surf B Biointerfaces. 2018;169:233-241. doi:10.1016/j.colsurfb.2018.05.027

28. Corpe RS, Steflik DE, Whitehead RY, Wilson MD, Young TR, Jaramillo C. Correlative experimental animal and human clinical retrieval evaluations of hydroxyapatite (HA)-coated and non-coated implants in orthopaedics and dentistry. Crit Rev Biomed Eng. 2000;28(3-4):395-398. doi:10.1615/CritRevBiomedEng.v28.i34.80

29. Wong MH, Man HC. Low-temperature fabrication of Ag-doped HA coating on NiTi. Mater Lett. 2018;229:229-231. doi:10.1016/j. matlet.2018.07.003

30. Terranova L, Dragusin DM, Mallet R, et al. Repair of calvarial bone defects in mice using electrospun polystyrene scaffolds combined with beta-TCP or gold nanoparticles. Micron. 2017;93:29-37. doi:10.1016/j.micron.2016.11.001

31. Singh AV, Jungnickel H, Leibrock L, et al. ToF-SIMS 3D imaging unveils important insights on the cellular microenvironment during biomineralization of gold nanostructures,". Sci Rep. 2020;10(1):261 doi:10.1038/s41598-019-57136-w
32. Singh AV, Alapan Y, Jahnke T, et al. Seed-mediated synthesis of plasmonic gold nanoribbons using cancer cells for hyperthermia applications. J Mater Chem B. 2018;6(46):7573-7581. doi:10.1039/ c8tb02239a

33. Choi SY, Song MS, Ryu PD, Lam ATN, Joo SW, Lee SY. Gold nanoparticles promote osteogenic differentiation in human adipose-derived mesenchymal stem cells through the Wnt/beta-catenin signaling pathway. Int J Nanomed. 2015;10:4383-4392. doi:10. 2147/ijn.s78775

34. Li JC, Li JJ, Zhang J, Wang XL, Kawazoe N, Chen GP. Gold nanoparticle size and shape influence on osteogenesis of mesenchymal stem cells. Nanoscale. 2016;8(15):7992-8007. doi:10.1039/ c5nr08808a

35. Deng J, Zheng H, Zheng X, Yao M, Li Z, Gao C. Gold nanoparticles with surface-anchored chiral poly(acryloyl-L(D)-valine) induce differential response on mesenchymal stem cell osteogenesis. Nano Res. 2016;9(12):3683-3694. doi:10.1007/s12274-016-1239-y

36. Yi CQ, Liu DD, Fong CC, Zhang JC, Yang MS. Gold nanoparticles promote osteogenic differentiation of mesenchymal stem cells through p38 MAPK pathway. Acs Nano. 2010;4(11):6439-6448. doi:10.1021/nn101373r

37. Zhang D, Liu D, Zhang J, Fong C, Yang M. Gold nanoparticles stimulate differentiation and mineralization of primary osteoblasts through the ERK/MAPK signaling pathway. Mater Sci Eng C. 2014;42:70-77. doi:10.1016/j.msec.2014.04.042

38. Heo DN, Ko W-K, Lee HR, et al. Titanium dental implants surface-immobilized with gold nanoparticles as osteoinductive agents for rapid osseointegration. $J$ Colloid Interface Sci. 2016;469: 129-137. doi:10.1016/j.jcis.2016.02.022

39. Singh AV, Batuwangala M, Mundra R, et al. Biomineralized anisotropic gold microplate-macrophage interactions reveal frustrated phagocytosis-like phenomenon: a novel paclitaxel drug delivery vehicle. ACS Appl Mater Interfaces. 2014;6(16):14679-14689. doi:10.1021/am504051b

40. Singh AV, Jahnke T, Kishore V, et al. Cancer cells biomineralize ionic gold into nanoparticles-microplates via secreting defense proteins with specific gold-binding peptides. Acta Biomaterialia. 2018;71: 61-71. doi:10.1016/j.actbio.2018.02.022

41. Gao TL, Zhang N, Wang Z, et al. Biodegradable microcarriers of poly(Lactide-co-Glycolide) and nano-hydroxyapatite decorated with IGF-1 via polydopamine coating for enhancing cell proliferation and osteogenic differentiation. Macromol Biosci. 2015;15(8):1070-1080. doi:10.1002/mabi.201500069

42. Singh AV, Sitti M. Patterned and specific attachment of bacteria on biohybrid bacteria-driven microswimmers. Adv Healthcare Mater. 2016;5(18):2325-2331. doi:10.1002/adhm.201600155

43. Tejido-Rastrilla R, Baldi G, Boccaccini AR. Ag containing polydopamine coating on a melt-derived bioactive glass-ceramic: effect on surface reactivity. Ceramics Int. 2018;44(13):16083-16087. doi:10. 1016/j.ceramint.2018.05.198

44. Shi H, Ye X, He F, Ye J. Improving osteogenesis of calcium phosphate bone cement by incorporating with lysine: an in vitro study. Coll Surf B Biointerfaces. 2019;177:462-469. doi:10.1016/j.colsurfb.2019.02. 034

45. Zhang J, Li J, Jia G, et al. Improving osteogenesis of PLGA/HA porous scaffolds based on dual delivery of BMP-2 and IGF-1 via a polydopamine coating. $R S C A d v$. 2017;7(89):56732-56742. doi:10. 1039/c7ra12062a

46. Luo Y, Shen H, Fang Y, et al. Enhanced proliferation and osteogenic differentiation of mesenchymal stem cells on graphene oxide-incorporated electrospun poly(lactic-co-glycolic acid) nanofibrous mats. ACS Appl Mater Interfaces. 2015;7(11):6331-6339. doi:10.1021/acsami.5b00862

47. Liu YM, Wen J, Gao Y, et al. Antibacterial graphene oxide coatings on polymer substrate. Appl Surf Sci. 2018;436:624-630. doi:10.1016/ j.apsusc.2017.12.006 
48. Burygin GL, Khlebtsov BN, Shantrokha AN, Dykman LA, Bogatyrev VA, Khlebtsov NG. On the enhanced antibacterial activity of antibiotics mixed with gold nanoparticles. Nanoscale Res Lett. 2009;4(8):794-801. doi:10.1007/s11671-009-9316-8

49. You D, Li K, Guo WL, Zhao GQ, Fu C. Poly (lactic-co-glycolic acid)/graphene oxide composites combined with electrical stimulation in wound healing: preparation and characterization. Int J Nanomedicine. 2019;14:7039-7052. doi:10.2147/ijn.s216365

50. Zhao Y, Jiang X. Multiple strategies to activate gold nanoparticles as antibiotics. Nanoscale. 2013;5(18):8340-8350. doi:10.1039/c3nr01990j

51. Zawrah MF, Abd El-Moez SI. Antimicrobial activities of gold nanoparticles against major foodborne pathogens. Life Sci J. 2011;8(4):37-44.

52. Tu Y, Lv M, Xiu P, et al. Destructive extraction of phospholipids from Escherichia coli membranes by graphene nanosheets. Nat Nanotechnol. 2013;8(8):594-601. doi:10.1038/nnano.2013.125

53. Krishnamoorthy K, Veerapandian M, Zhang L-H, Yun K, Kim SJ. Antibacterial efficiency of graphene nanosheets against pathogenic bacteria via lipid peroxidation. $J$ Phys Chem C. 2012;116 (32):17280-17287. doi:10.1021/jp3047054

54. Yan J, Wu RY, Liao SS, Jiang M, Qian Y. Applications of polydopamine-modified scaffolds in the peripheral nerve tissue engineering. Front Bioengineering Biotechnol. 2020;8:590998. doi:10.3389/fbioe. 2020.590998

55. Tsai WB, Chen WT, Chien HW, Kuo WH, Wang MJ. Poly(dopamine) coating to biodegradable polymers for bone tissue engineering. J Biomater Appl. 2014;28(6):837-848. doi:10.1177/0885328213483842

56. Ryu J, Ku SH, Lee H, Park CB. Mussel-inspired polydopamine coating as a universal route to hydroxyapatite crystallization. Adv Funct Mater. 2010;20(13):2132-2139. doi:10.1002/adfm.200902347
57. Tetsunaga T, Nishida K, Furumatsu T, et al. Regulation of mechanical stress-induced MMP-13 and ADAMTS-5 expression by RUNX-2 transcriptional factor in SW1353 chondrocyte-like cells. Osteoarthritis Cartilage. 2011;19(2):222-232. doi:10.1016/j.joca.20 10.11.004

58. Singh AV, Dad Ansari MH, Dayan CB, et al. Multifunctional magnetic hairbot for untethered osteogenesis, ultrasound contrast imaging and drug delivery. Biomaterials. 2019;219:119394. doi:10.1016/j. biomaterials.2019.119394

59. Dalla-Costa K, Yurtsever FV, Penteado J, Martinez EF, Sperandio M, Peruzzo DC. Melatonin has a stimulatory effect on osteoblasts by upregulating col-i and opn expression/secretion. Acta Odontologica Latinoamericana. 2020;33(2):125.

60. Dinescu S, Ionita M, Ignat SR, Costache M, Hermenean A. Graphene oxide enhances chitosan-based 3D Scaffold properties for bone tissue engineering. Int J Mol Sci. 2019;20(20):5077. doi:10.3390/ijms20 205077

61. Lee JH, Shin YC, Lee S-M, et al. Enhanced osteogenesis by reduced graphene oxide/hydroxyapatite nanocomposites. Sci Rep. 2015;5 (1):13. doi: $10.1038 /$ srep 18833

62. Niu C, Yuan K, Ma R, et al. Gold nanoparticles promote osteogenic differentiation of human periodontal ligament stem cells via the p38 MAPK signaling pathway. Mol Med Rep. 2017;16(4):4879-4886. doi: $10.3892 / \mathrm{mmr} .2017 .7170$
International Journal of Nanomedicine

\section{Publish your work in this journal}

The International Journal of Nanomedicine is an international, peerreviewed journal focusing on the application of nanotechnology in diagnostics, therapeutics, and drug delivery systems throughout the biomedical field. This journal is indexed on PubMed Central, MedLine, CAS, SciSearch ${ }^{\mathbb{}}$, Current Contents ${ }^{\mathbb{R}} /$ Clinical Medicine, $^{-}$

\section{Dovepress}

Journal Citation Reports/Science Edition, EMBase, Scopus and the Elsevier Bibliographic databases. The manuscript management system is completely online and includes a very quick and fair peer-review system, which is all easy to use. Visit http://www.dovepress.com/ testimonials.php to read real quotes from published authors. 